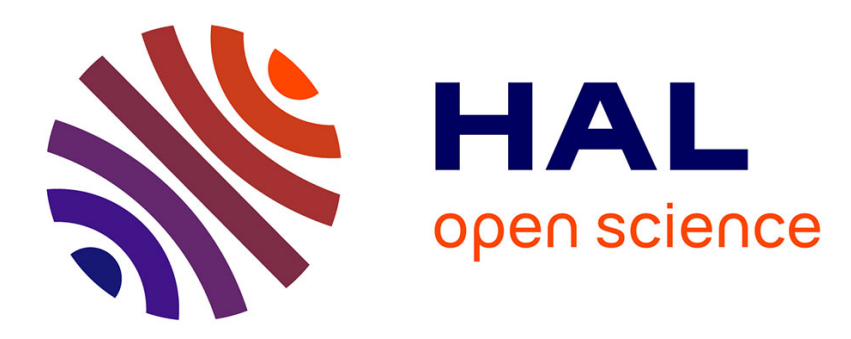

\title{
Non-linear vibrations of shells: A literature review from 2003 to 2013
}

Farbod Alijani, Marco Amabili

\section{To cite this version:}

Farbod Alijani, Marco Amabili. Non-linear vibrations of shells: A literature review from 2003 to 2013. International Journal of Non-Linear Mechanics, 2014, 10.1016/j.ijnonlinmec.2013.09.012 . hal01299631

\section{HAL Id: hal-01299631 \\ https://hal.science/hal-01299631}

Submitted on 8 Apr 2016

HAL is a multi-disciplinary open access archive for the deposit and dissemination of scientific research documents, whether they are published or not. The documents may come from teaching and research institutions in France or abroad, or from public or private research centers.
L'archive ouverte pluridisciplinaire HAL, est destinée au dépôt et à la diffusion de documents scientifiques de niveau recherche, publiés ou non, émanant des établissements d'enseignement et de recherche français ou étrangers, des laboratoires publics ou privés. 


\title{
Non-linear vibrations of shells: A literature review from 2003 to 2013
}

\author{
Farbod Alijani, Marco Amabili
}

Department of Mechanical Engineering, McGill University, 817 Sherbrooke Street West, Montreal, Canada H3A 0C3

The present literature review focuses on geometrically non linear free and forced vibrations of shells made of traditional and advanced materials. Flat and imperfect plates and membranes are excluded. Closed shells and curved panels made of isotropic, laminated composite, piezoelectric, functionally graded and hyperelastic materials are reviewed and great attention is given to non linear vibrations of shells subjected to normal and in plane excitations. Theoretical, numerical and experimental studies dealing with particular dynamical problems involving parametric vibrations, stability, dynamic buckling, non stationary vibrations and chaotic vibrations are also addressed. Moreover, several original aspects of non linear vibrations of shells and panels, including (i) fluid structure interactions, (ii) geometric imperfections, (iii) effect of geometry and boundary conditions, (iv) thermal loads, (v) electrical loads and (vi) reduced order models and their accuracy including perturbation techniques, proper orthogonal decomposition, non linear normal modes and meshless methods are reviewed in depth. 


\section{Introduction}

Shell structures are used in aircraft, spacecraft, rockets, cars, computers, submarines, boats, storage tanks and the roofs of buildings. In the last decade, the continuous development of material science and engineering, along with the increasing demand to produce light weight structures, has led to the use of advanced materials (laminated composites and functionally graded materials (FGMs)) in designing shell structures. Among diverse fields of application, aerospace and aeronautical applications are particularly challenging as they involve fluid structure interactions and the use of new materials with less known properties. Therefore, models should be developed that take into account non linear effects, such as large structural deflections, and predictions of the structural responses to large amplitude excitations.

In case of linear vibrations of closed (circumferentially complete) circular cylindrical shells subjected to radial periodic excitations, the mode shape of the shell is a standing wave directly excited by the external excitation, which presents a number $n$ of nodal diameters. This is known as the driven mode. For large amplitude vibrations of shells there is an intrinsic one to one internal resonance between the driven mode and the orthogonal mode having the same shape and natural frequency as the driven mode, but rotated by $\pi / 2 n$, known as the companion mode. This mode interaction yields a traveling wave response in the circumferential direction of the shell that appears even for vibration amplitudes smaller than the shell thickness. In fact, the presence of a traveling wave response near resonance has a fundamental difference with respect to linear vibrations. Another important feature of closed circular cylindrical shells in large amplitude vibrations is the dynamic axisymmetric contraction, which has been addressed both theoretically and experimentally. In particular, during large amplitude vibrations, the shell presents an inward axisymmetric dynamic contraction with twice the excitation frequency to guarantee the in plane quasi inextensibility of the shell. Without this axisymmetric con traction, the shell would increase its length along the circumference significantly, which is against shell mechanics. In fact, shells bend more easily than they stretch.

Shell structures subjected to in plane loads lose stability and buckle after a certain threshold. In fact, in the presence of static in plane loads, instability occurs through pitchfork bifurcation, whereas in the case of periodic in plane loads the so called parametric instability occurs through period doubling bifurcation in the frequency neighbor hood of twice the natural frequencies of the bending mode. In the latter case, and in contrast with non linear vibrations of shells due to radial harmonic excitation, the instability may arise even for small excitation amplitudes and much below the static buckling load.

Shell structures that are coupled to a liquid have lower natural frequencies as a consequence of the added virtual mass of the liquid itself. In closed circular cylindrical shells, the effect of added virtual mass is lower for the asymmetric modes than for axisym metric modes. Therefore, the non linearity displayed by thin closed circular shells is enhanced by the presence of dense fluid. Moreover, circular cylindrical shells supported or clamped at both ends and conveying liquid lose stability via divergence, which is a static pitchfork bifurcation of the equilibrium, when the flow speed reaches a critical value. The divergence is strongly subcri tical, becoming supercritical for high flow velocities. In this case, the system has two or more stable solutions related to divergence much before pitchfork bifurcation occurs. In other words, if the shell is perturbed from the initial configuration, it may have severe deformations resulting in failure much below the critical velocity that can be predicted by linear analysis.

Complicated dynamics and chaotic motions are other interest ing features that have been widely reported for shell structures. These complicated motions may occur for shells subjected to large amplitude excitations, at specific excitation frequencies and force amplitudes.
Shell structures generally do not follow a certain scenario to become chaotic, since the routes to chaos depend on all system parameters. These complex behaviors are usually analyzed using bifurcation diagrams, Poincaré maps, phase plane plots, time responses and spectra, Lyapunov exponents, Lyapunov spectrum and fractal dimensions.

In contrast with closed circular cylindrical shells, curved panels (i.e. open shells, with single and double curvature) do not always exhibit an internal resonance, unless for a specific shallowness and thickness ratios, and do not have axisymmetric dynamic contrac tion. The peculiar aspects of panels that make the non linear analysis of these structures interesting are their asymmetric nature of oscillation with respect to the initial un deformed middle surface, the softening non linear behavior that turns into hardening for large amplitude vibrations and the diverse internal resonance conditions that may occur for different geometries.

The present paper aims to review the existing literature on non linear vibrations of shell structures from 2003 to 2013. Flat and imperfect plates and membranes are excluded. Literature reviews of the earlier works on large amplitude vibrations of shells before 2003 include those of Leissa [1], Evensen [2], Kubenko and Koval'chuk [3,4], Amabili and Païdoussis [5] and Moussaoui and Benamar [6]. In fact, an extensive literature review until 2003 on the non linear dynamics of shells in vacuo, filled with or surrounded by quiescent and flowing fluids, has been provided in Ref. [5] and will not be repeated here. However, to elaborate the new research carried out on non linear vibrations of shell type structures, it is necessary to refer to some fundamental references, briefly. The monograph of Amabili [7] has also provided a literature review on non linear vibrations and dynamic stability of shells till 2008 and has shown that many questions in this area still remain unan swered. Therefore, the present literature review focuses on geome trically non linear free and forced vibrations of closed and open shells made from traditional and advanced materials with and without fluid structure interaction. Studies dealing with particular dynamical problems involving parametric vibrations, stability, dynamic buckling, non stationary vibrations and chaotic vibrations are profoundly addressed. Moreover, several original aspects of non linear vibrations of shells and panels, including geometric imperfections, effect of geometry and boundary conditions, thermal loads, electrical loads and reduced order models including pertur bation techniques, proper orthogonal decomposition, non linear normal modes, natural modes and meshless methods, are fully reviewed. Works dealing with experiments are also discussed.

The reviews are generally organized chronologically, according to the publication date, and are structured as follows: non linear theories of shell structures are discussed in Section 2. In Section 3, non linear vibrations of circumferentially complete shells are given. In order to better elaborate the review, Section 3 has been divided into four subsections depending on the type of loads applied on the shell. In particular, in Section 3.1, free and forced vibrations of closed isotropic shells due to radial harmonic excita tion are studied; in Section 3.2, dynamics and stability of closed shells subjected to axial loads are discussed, and in Section 3.3 the existing literature of shells conveying fluid are given. Section 3.3 has been divided into two subsections, Section 3.3.1 deals with dynamical problems of shells filled with still fluid and Section 3.3.2 discusses the stability and dynamics of shells subjected to flowing fluid. In Section 3.4, papers dealing with non linear vibrations of closed isotropic shells subjected to non stationary, thermal and electrical forces are reviewed.

In Section 4, non linear free and forced vibrations of curved panels are discussed. Depending on the geometry of the panel, this section has been divided into 2 subsections: Section 4.1 discusses cylindrical and doubly curved panels and Section 4.2 discusses spherical caps and panels of other geometries including conical and ellipsoidal shapes.

Non linear vibrations of shells made of advanced materials are discussed in Sections 5 and 6. In particular, sandwich and 
laminated composite shells, panels and fluid structure interaction in composite shells are reviewed in Sections 5.1, 5.2 and 5.3, respectively, and literature on non linear vibrations of FGM shells and panels can be found in Sections 6.1 and 6.2, respectively. Section 7 is dedicated to non linear vibrations of hyperelastic shells and, finally, papers dealing with experiments are listed in Section 8.

\section{Non-linear theories of shells}

The classical theories of non linear shell mechanics, suitable for thin shells, include those of Donnell [8], Novozhilov [9], Sanders [10], Koiter [11] and Flügge Lur'e Byrne [12] that are all based on the Kirchhoff Love hypotheses. In particular, Donnell's non linear shallow shell theory neglects in plane inertia and gives accurate results only for very thin shells. In this theory, in plane displace ments are infinitesimal, while the transverse displacement is of the order of shell thickness. Moreover, the non linear terms are retained only in the transverse displacement (the von Kármán type non linear terms, in analogy to the treatment of plates); however, other effects, such as the non linearities involving in plane displacements, are neglected. The Donnell theory, without the shallow shell hypothesis, retains in plane inertia and is more accurate than Donnell's non linear shallow shell theory; however, it still keeps non linear terms of only the von Kármán type. The Sanders theory is a more refined non linear shell theory devel oped by Sanders [10] originally in tensorial form. The same theory was also derived independently by Koiter [11] around the same period, leading to the designation of this theory as the Sanders Koiter non linear shell theory. This theory is suitable for finite deformations with small strain and moderately small rotations. Therefore, in the Sanders Koiter theory the in plane displace ments are not infinitesimal, and in the strain displacement rela tions, non linear terms that depend on both in plane and transverse displacements exist. In both the Donnell and the Sanders Koiter theories the changes of curvature and torsion of the middle surface are assumed to be linear. Moreover, the Sanders Koiter theory gives accurate results for vibration ampli tudes significantly larger than the shell thickness, whereas Don nell's theory is accurate only for very thin shells and modes of high circumferential wave number.

The general non linear theories of shells developed by Novoz hilov [9] and the Flügge Lur'e Byrne theory [12] are very similar and only differ in terms of change in curvature and torsion. In both of these theories, non linearities can be retained in changes in curvature and torsion [7]. Moreover, the thinness assumption is delayed in the derivations of both theories and the strain displacement relations include non linearities in both transverse and in plane displacements. All of these classical theories are obtained by neglecting transverse shear deformation and rotary inertia, and thus, can give inaccurate results for moderately thick or laminated anisotropic shells. In order to overcome this limita tion, shear deformation theories have been introduced. These theories can be classified as the first order and higher order shear deformation theories; in the first category, a shear correction factor is required for the equilibrium since a uniform shear strain is assumed through the shell thickness. Higher order shear defor mation theories overcome this limitation since a realistic shear stress distribution through the shell thickness is assumed, which also satisfies the condition of zero shear stresses at both the top and bottom shell surfaces. These theories have been completely discussed in the books of Amabili [7], Reddy [13] and Carrera et al. [14]. Moreover, linear shear deformable and zigzag (layer wise constant shear angle) theories have been profoundly reviewed by Carrera [15,16] and Reddy and Arciniega [17].
The non linear first order shear deformation theory was pri marily proposed by Reddy and Chandrashekhara [18] and was based on Sanders Koiter non linear terms. Five independent variables three displacements and two rotations were used to describe the shell deformations. Non linear first order shear deformation theory of shells based on von Kármán type non linear terms and its finite element formulations is also given in the study by Reddy [13]. Librescu [19] developed a non linear shell theory by expanding the shell displacements with cubic terms in the transverse coordinate. Dennis and Palazotto [20] and Palazotto and Dennis [21] extended Reddy's linear higher order shear deformation shell theory to non linear deformation by introducing the von Kármán type non linear terms. The non linear higher order shell theory of sandwich anisotropic shells with transversely compressible core was developed by Hohe and Librescu [22]. In their theory, the Kirchhoff Love hypotheses were adopted for the face sheets and a second/ third order power series expansion was considered for the core displacements. Chaudhuri [23] pro posed a non linear zigzag theory for the non linear finite element analysis of doubly curved shells by considering the full non linear strain displacement relations for the five independent variables of the shell. Amabili and Reddy [24] developed a new theory for closed and open shells that, in contrast with previous non linear shell theories, was derived with consistency retaining rotary inertia, shear deformation and non linearities in both in plane and transverse displacements. The new theory has shown super iorities over the existing non linear shear deformation theories in predicting large amplitude vibrations of deep and moderately thick laminated circular cylindrical shells [25] and curved panels [26]. Recently, Amabili [27] extended the theory of [24] by adding the thickness stretching effect and taking into account the geo metric imperfections. In this new theory, six independent vari ables were used to describe shell deformation. In particular, in [27] a uniform transverse normal strain is assumed through the shell thickness. This theory has been extended to third order transverse normal strain by Amabili [28]. The advantages of shell theories retaining transverse normal stress and strain are using the full three dimensional constitutive equations and they are particularly suitable for soft materials, like rubbers and biological materials, where very large deformations are achieved and accompanied by large thickness reduction. Parisch [29] and Sansour [30] developed independently shell theories that introduce quadratic assumption of shell displacement over shell thickness. Moreover, an accurate linear shell theory that takes into account the thickness variation was developed by Carrera et al. [31] and Ferreira et al. [32]. Tensor based geometrically non linear theories of shells are quite abun dant in the literature [33 45]. Eremeyev and Pietraszkiewicz [33] developed a general non linear theory for shells taking into account the phase transitions of materials. In their theory shell displacements were expressed by work averaged translations and rotations of the shell cross sections. Moreover, all shell relations were found from the variational principle of the stationary potential energy. Opoka and Pietraszkiewicz [34] consistently obtained a complete boundary value problem for the non linear theory of thin elastic shells expressed thoroughly in terms of stress resultants and bending of the shell reference surface. Arciniega and Reddy [35] developed an improved first order shear deforma tion theory with seven independent variables under the Lagran gian framework and presented the finite element formulations that could be used to study the non linear analysis of a broad range of shell geometries made of different types of materials including isotropic, laminated composite and FGMs. Opoka and Pietraszkiewicz [36] modified their previous work [34] by for mulating a new version of the Lagrangian theory of thin shells in terms of displacements of the reference surface and based on the principle of virtual work. Berdichevsky [37] proposed a non linear 
theory for sandwich shells based on the asymptotic variational method. Shen et al. [38] developed a modified Koiter shell theory and discussed the role of metric and curvature tensors in con structing non linear elastic shell models of the Koiter type. Pietraszkiewicz [39] reviewed some equivalent expressions of the bending tensor in the non linear shell theory and indicated that the one proposed by Shen et al. [38] was not exact. The Koiter shell theory has also been discussed from the perspective of non linear three dimensional elasticity by Steigmann [40].

Non linear shell theories that were proposed using the Cosserat continuum can be found in Refs. [41 45]. The basic idea of this variant of continuum mechanics model is the independence of translations and rotations, and therefore by analogy the indepen dence of forces and moments. The non linear theory of micropolar shells has been given in Ref. [41], and the local symmetry group for the dynamical exact theory of shells is established in Ref. [42]. A short review of Cosserat theories for shell structures was carried out by Altenbach et al. [44], and the non linear shell theory in nanoscale was presented by Altenbach and Ermeyev [45]. Moreover, the non linear strain gradient theory of shells was presented by Lazopoulos and Lazopoulos [46].

\section{Non-linear vibrations of closed isotropic shells}

This section is divided into four parts, depending on the type of excitation: (i) free and forced vibrations of shells due to radial excitation; (ii) dynamics and stability of shells subjected to axial loads; (iii) fluid shell interaction; and (iv) forced vibrations of shells subjected to other types of loading conditions.

\subsection{Free and forced vibrations of shells due to radial excitation}

The history of thin circular cylindrical shells subjected to large amplitude excitations and discussions made by different researchers on the softening nature of the response has been well explained in the review paper of Amabili and Païdoussis [5]. However, it is worth having a brief review on how the correct behavior of shells can be numerically modeled. In fact, to accurately predict the large amplitude behavior of circular cylindrical shells, the mode shapes should be expanded by sufficient asymmetric and axisymmetric modes. The original idea of mode expansions of shell flexural displacement involving the driven and companion modes plus an axisymmetric term is attributed to Evensen [2,47,48], Dowell and Ventres [49] and Atluri [50]. In particular, the axisymmetric term considered by Evensen was a squared sine in the axial coordinate with the amplitude related to the amplitude of linear terms in order to satisfy the continuity of the shell circumferential displacement, while Refs. [49,50] only considered the first axisymmetric mode of linear vibrations in order to satisfy exactly the out of plane boundary conditions and the in plane boundary conditions on average. Although the effect of adding an axisymmetric term in the mode expansion was found to be significant, discrepancies still existed between theory and experiments since experimental observations of Evensen [51] and Olson [52] illustrated softening type non linearity whereas the numerical results of Dowell and Venters [49] and Atluri [50] predicted hardening non linearity. Varadan et al. [53] showed that the hardening type results obtained in Refs. $[49,50]$ are due to the choice of the axisymmetric term. Amabili $[54,55]$ elaborated the effect of axisymmetric terms and showed that in order to correctly predict the softening behavior of circular cylindrical shells, at least the first two axisymmetric modes (axisym metric modes with an even number of longitudinal half waves do not give any contribution) should be retained in the mode expansion in addition to the driven and companion modes.

In 2003, Amabili [56,57] extended his previous works on non linear forced vibrations of simply supported circular cylindrical shells subjected to concentrated harmonic force. In particular, in Ref. [56], Donnell's non linear shallow shell theory was used and the non linear frequency response curves were obtained using the Galerkin method considering several expansions, including 16 or more natural modes of the shell that included sufficient asymmetric and axisymmetric modes. Moreover, a code based on the arc length continuation technique was utilized to perform bifurcation analysis. Several interesting non linear phenomena were observed including softening type non linearity, different types of traveling wave response, amplitude modulated response and modal interactions. In order to validate the numerical simula tions and to understand the physical behavior of the shell, experiments were also carried out following a stepped sine technique and by spanning the excitation frequency up and down in very small increments in the frequency neighborhood of the shell's fundamental frequency. In the experimental tests the response of the shell was measured by using two accelerometers and the excitation was provided by an electrodynamic exciter connected to the shell by a stinger glued in a position close to the middle of the shell. The time responses were measured using a Difa Scadas II front end connected to a workstation with the software CADA X of LMS for signal processing. The same front end was used to generate the excitation and its closed loop control was used to keep the value of the excitation force constant for any excitation frequency, during the measurements of non linear response.

Amabili [57] compared the numerical results obtained from Donnell's non linear theory (with and without in plane inertia) with Sanders Koiter, Flügge Lur'e Byrne and Novozhilov's theories following a Lagrangian approach. Different expansions involving 1448 generalized coordinates, associated with natural modes of simply supported shells, were used and it was shown that the results from Sanders Koiter, Flügge Lur'e Byrne and Novozhilov are practically coincident. Moreover, a small difference was observed between the previous three theories and Donnell's theory with in plane inertia. On the other hand, Donnell's non linear shallow shell theory proved to be the least accurate among the five theories compared. Comparisons between these theories in predicting the non linear response of a circular cylindrical shell are shown in Fig. 1.

The internal resonances of undamped and unloaded circular cylindrical shells were investigated by Popov [58,59] by means of Hamiltonian dynamics. The analysis was based on Donnell's shell theory and the Rayleigh Ritz technique was used to discretize the equations of motion and to study non linear systems that had two and three degrees of freedom, respectively. Avramov [60] and Avramov et al. [61] used the method of multiple scales to study the forced vibrations of circular cylindrical shells with two internal resonances and found hardening behavior for standing and traveling wave responses of the shell. They used Donnell's non linear theory and an expansion for the transverse displacement that consisted of only the fundamental driven and companion modes plus an axisymmetric term in the form of a squared sine in the axial coordinate. In fact, the source of the reported hardening non linearity could be due to the inappropriate expansions considered for the flexural displacement.

To study non linear forced vibrations of simply supported shells, Gonçalves et al. [62] presented a low dimensional model, combining a perturbation technique with the proper orthogonal decomposition (POD) method. In their analysis, the POD method was used to investigate the relative importance of each mode obtained by the perturbation solution on the non linear response and the total energy of the system. Touzé et al. [63] used the non linear normal modes method to predict the response of circular cylindrical shells subjected to harmonic excitation and compared the accuracy of different truncations obtained using linear and non linear modes. They showed that a reduced order model comprising one or two 


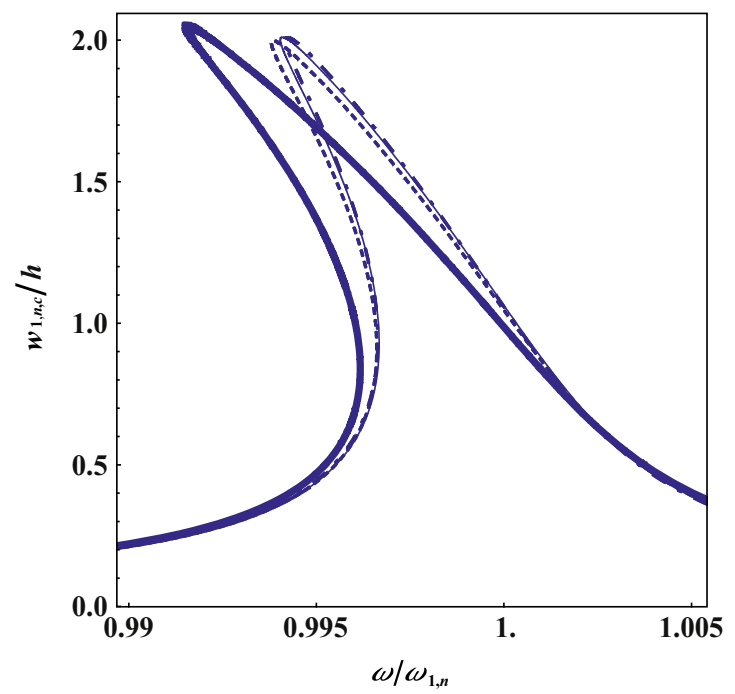

Fig. 1. Non-linear frequency response curve for the fundamental mode of a perfect shell subjected to radial harmonic load. $\omega_{1, n}$ is the fundamental frequency, $\omega$ is the excitation frequency, $w_{1, n, c}$ is the amplitude of the driven mode, $h$ denotes the thickness. Donnell's shallow shell theory without in-plane inertia, Donnell's theory with in-plane inertia, Sanders-Koiter theory, Flugge-Lu're-Byrne theory (coincident with Novozhilov). From Amabili [57].

non linear normal modes gives perfect results for vibration ampli tudes lower than or equal to the thickness of the shell. However, non linear normal modes obtained by perturbation techniques, as other perturbation techniques, lose accuracy when the vibration amplitude becomes larger.

Using a Lagrangian approach and the method of harmonic balance, Rougui et al. [64] investigated the non linear free and forced vibrations of simply supported cylindrical shells. A simple approximate transverse displacement expansion including the driven mode and the axisymmetric modes (neglecting the com panion modes and using the squared sin axisymmetric term) was used for the minimization of the energy functional and it was shown that the shell exhibits a softening behavior. The method of harmonic balance was also successfully used by Avramov [65,66] in obtaining the non linear normal modes and traveling waves of simply supported circular cylindrical shells.

Non linear free and forced vibrations of rotating shells were investigated by Chen and Dai [67], Wang et al. [68 70], Liu and Chu [71] and Chen [72]. In particular, in Refs. [67,72] the method of multiple scales was used to study the non linear vibrations of rotary truncated conical shells with simply supported and clamped bound ary conditions, and it was found that the shell exhibits hardening behavior. Using Donnell's non linear shallow shell theory, Wang et al. $[68,69]$ used different multi dimensional models and included the Coriolis force and damping effects in the equations of motion. To obtain the frequency response curves, the equations of motion were solved by direct time integration in [68] and the method of harmonic balance in [69]. Wang et al. [70] also studied the non linear vibrations of cantilevered shells subjected to a moving concentrated harmonic force along the shell using the harmonic balance method. Liu and Chu [71] used a single mode Galerkin approximation together with the method of multiple scales to obtain the fre quency response curves of cantilevered rotating thin circular cylind rical shells. They used the eigen functions of a clamped free beam to discretize the equations of motion. In all these studies, Refs. [67 72], the effect of axisymmetric modes was neglected and therefore hardening behavior was obtained for the shells under investigation.

Kurylov and Amabili [73] compared the large amplitude response of isotropic shells obtained using Chebyshev polynomials, power polynomials and trigonometric functions for simply supported and clamped boundary conditions. The Sanders Koiter non linear shell theory was used and it was found that trigonometric functions are very efficient for simply supported boundary conditions while they require a large number of degrees of freedom for other boundary conditions, thereby decreasing the computational efficiency. It was also found that Chebyshev polynomials have the advantage of requiring similar degrees of freedom for any boundary condition whereas ordinary power polynomials present ill conditioned mass and stiffness matrices in the linear problem, which can become problematic in non linear analysis. The same authors [74] also thoroughly investigated the non linear vibrations of cantilevered circular cylindrical shells using the Sanders Koiter non linear shell theory and expanded the shell displacements in terms of a harmonic function for the circumferential variable and Chebyshev polynomials for the longitudinal variable. A novel technique to reduce the number of degrees of freedom by introducing artificial axisymmetric modes in the expansions of the displacements was used and different expansions involving 1852 generalized coordinates were considered to study the convergence of the solution.

Non linear vibrations of cantilevered shells were also studied by Zhang et al. [75]. The shell was assumed to be in high speed milling process and was subjected to a harmonic transverse cutting force. Considering a two to one internal resonance between the first two asymmetric modes of the shell and neglecting the companion and axisymmetric modes, the method of multiple scales was used to obtain the modulation equations and the fourth order Runge Kutta algorithm was utilized to solve the modulation equations and to obtain the bifurcation diagrams.

Non linear free vibrations of pre stressed circular cylindrical shells having combinations of clamped, free and simply supported boundary conditions, resting on an elastic foundation, were investigated by Bakhtiari Nejad and Bideleh [76]. The non linear Sanders Koiter theory was used and a minimal single mode approximation with the Lindstedt Poincaré perturbation technique was utilized to obtain the backbone curves that showed erroneous hardening behavior.

The effect of geometric imperfections on the large amplitude response of circular cylindrical shells was studied in Refs. $[56,57,61,74]$. In particular, Amabili $[56,57]$ discussed the effect of three different sets of imperfections on the fundamental mode of a simply supported shell, and showed that asymmetric imperfec tions having the same shape of the fundamental mode have a large effect on the non linear response of the shell. He showed that, due to the presence of small imperfection, the trend of non linearity is not affected while the traveling wave response is largely modified since the natural frequencies of the driven and companion modes do not coincide anymore. The initial geometric imperfection having the shape of the driven and companion modes of the shell was also taken into account by Avramov et al. [61]. They found that the non linear normal modes of the shell are mainly determined by shell imperfections. Kurylov and Amabili [74] studied the effect of imperfection on large amplitude vibrations of clamped free shells in depth and found that unlike other types of boundary conditions, geometric imperfections introducing an ovalization of the shell play an important role in non linear response, increasing significantly the softening behavior of the shell.

Chaotic vibrations of circular cylindrical shells were studied in Refs. [77 82]. In particular, using the Galerkin approach and the fourth order Runge Kutta method, Krysko et al. [77] showed that the chaotic regions of a circular cylindrical shell depend greatly on the loading angle when the shell is subjected to transversal sinusoidal load. Moreover, they investigated the local and global stability loss of closed shells and found that stiff stability loss is associated with a Hopf bifurcation and transition to chaotic and harmonic motions. Krysko et al. $[78,79]$ illustrated that increasing the amplitude of the external load in the presence of various 
temperature intensities can yield qualitative changes in the dynamics of the shell and can result in transition from chaotic behavior to harmonic and vice versa. In a series of papers, Awrejcewicz et al. [80], Krysko et al. [81] and Awrejcewicz et al. [82] discussed comprehensively the possible routes to chaos in circular cylindrical shells. In particular, in the first part [80], the practical convergence of diverse numerical methods including Galerkin, finite difference method, Ritz and different Runge Kutta techniques in predicting the chaotic behavior of shells was discussed. In the second part [81], it was shown that closed circular cylindrical shells may exhibit the modified Pomeau Manneville scenario and closed shells reinforced by flexible non compressed ribs may exhibit the modified Ruelle Takens New house Feigenbaum scenario to chaotic vibrations, and in the third part [82] the Lyapunov exponents were calculated.

Non linear vibrations of closed spherical shells were studied thoroughly by Arafat and Nayfeh [83]. The possibility of activation of two to one internal resonance between different vibration modes of the shell was investigated, and the method of multiple scales was used to obtain primary resonance responses and bifurcations due to an external axisymmetric excitation.

\subsection{Dynamics and stability of shells subjected to axial loads}

The parametric instability and the post critical behavior of simply supported circular cylindrical shells subjected to periodic axial loads were studied by Pellicano and Amabili [84]. Donnell's non linear shallow shell theory was used and the equations of motion were obtained using the Galerkin method and discretizing the governing partial differential equations with a relatively large number of linear modes, including axisymmetric and asymmetric modes. To perform bifurcation analysis, a pseudo arc length continuation scheme was utilized and the role of parametric resonance in the structural collapse of shells was confirmed. It was shown that, when the excitation frequency is close to twice the natural frequency of an asymmetric mode, a period doubling bifurcation takes place, result ing in quite large vibration amplitudes. Fig. 2(a c) shows the response of a thin perfect shell under periodic axial load. It can be clearly observed that dynamic instability occurs much below the static buckling load as a result of period doubling bifurcation. It can be seen that before the bifurcation point, only axisymmetric modes contribute to the vibration of the shell.

The parametric resonance of circular cylindrical shells has also been discussed by Popov [85], who considered a simple model of the shell with two degrees of freedom. In a series of papers, Gonçalves and del Prado [86,87] and Gonçalves et al. [88 90] studied the dynamic instability of axially excited simply supported, perfect circular cylindrical shells using Donnell's non linear shal low shell theory and following the Galerkin approach. In particular, modal interactions between non linear vibration modes with equal or nearly equal natural frequencies and their influence on para metric and snap through instability were studied in [86], and suitable reduced order models, which could reproduce the shell response accurately, were obtained in [87].Transient and steady state stability boundaries of an isotropic shell were obtained in [88] and special attention was devoted to the determination of critical loading conditions using a numerical continuation technique and the Runge Kutta method. Moreover, the complexity of the basins of attraction of low frequency vibration responses and the escape from the potential well as a result of combined static and periodic axial loads were clarified in [89]. In Ref. [90], the same authors focused more on the use of basins of attraction as a measure of reliability and safety of the shell and commented that, due to the fractal nature of the basin boundaries and as a result of the sub critical behavior, the shell response is very sensitive to initial conditions and uncertainties in system parameters. a

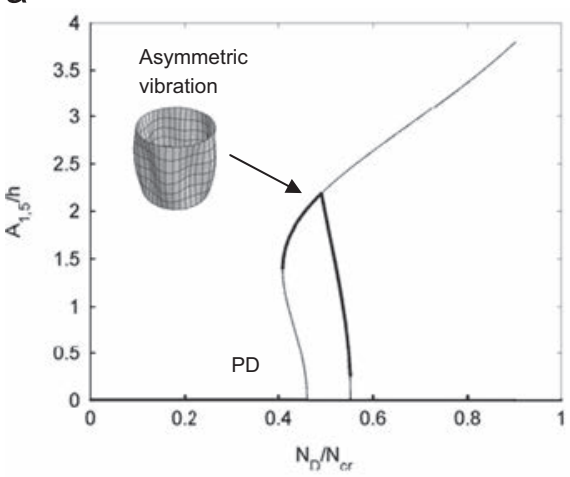

b

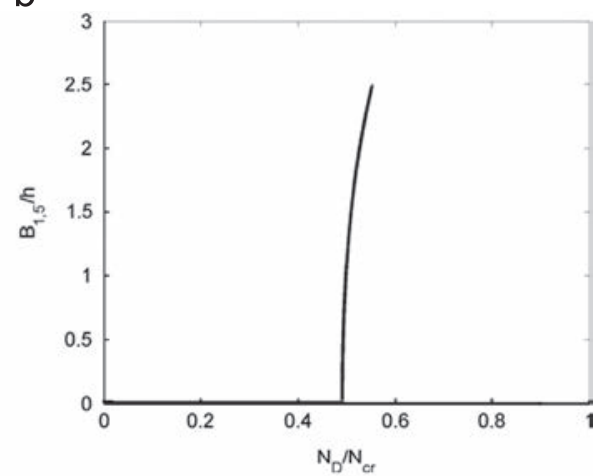

C

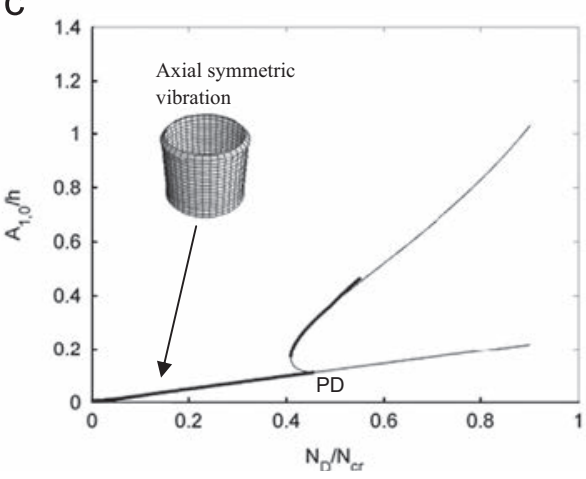

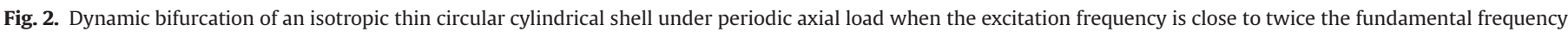

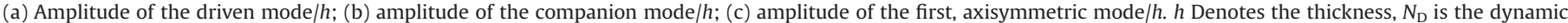
load, $N_{\mathrm{cr}}$ is the critical static buckling load and PD is period-doubling bifurcation. From Pellicano and Amabili [84]. 
Kochurov and Avramov [91] investigated the non linear modes and traveling waves of parametrically excited cylindrical shells. They used Donnell's non linear shallow shell theory and consid ered an eight degree of freedom model consisting of six asym metric modes, a squared sine term to describe the asymmetry of displacements with respect to the shell middle surface and a constant term that did not depend on the circumferential coordi nate. They mentioned that the constant term is added to describe the radial displacement of the points of the end sections of the shell or in other words to assume that the end sections can "breathe" during vibrations. Moreover, using the harmonic balance method, they found that non linear normal modes of vibration correspond to standing waves, and the behavior of non linear normal modes and traveling waves is qualitatively similar. The same authors [92] also studied the parametric resonance of circular cylindrical shells with a combination of resonance. A six degree of freedom model was considered. The modulation equa tions were derived for two types of combination of resonances by employing the method of multiple scales and it was shown that, depending on the combination of resonance, the shell exhibits either softening or hardening behavior.

By considering a two degree of freedom model based on Donnell's non linear shallow shell theory, Gonçalves et al. [93] studied the global dynamics of isotropic shells due to a combination of static and harmonic axial loads. By plotting Poincaré sections, bifurcation diagrams and basins of attractions, sub critical and super critical bifurcation scenarios were investigated and periodic, quasi periodic and chaotic motions were obtained.

Parametric stability of rotating truncated conical shells was investigated by Chen and Dai [67] and Chen [72]. The method of harmonic balance was used to obtain the regions of instability. Moreover, by considering a single degree of freedom model, Chen [72] used the Melinkov method to study the homoclinic and sub harmonic bifurcation of the shell, and the method of multiple scales to obtain the frequency amplitude curves.

Theory and experiments for non linear dynamics of circular cylindrical shells connected to a top mass and under base excitation were conducted by Pellicano and Avramov [94] and Pellicano $[95,96]$. In particular, in Ref. [94] dynamics of a shell carrying a rigid disk on top and clamped at the bottom was investigated using the Sanders Koiter non linear theory and solutions were obtained using a perturbation technique and direct time integration of equations of motion. Moreover, linear experimental modal analysis was performed and the damping ratios obtained from the experiments were used to conduct the non linear analysis. In Ref. [95], non linear vibrations were studied via a Lagrangian approach based on the Sanders Koiter theory, and Chebyshev polynomials were used to discretize the energy functional. The experimental analysis carried out in Ref. [95] was similar to that in Ref. [94] and included linear modal analysis. In Ref. [96], Pellicano extended his previous works by modifying the experimental analysis and obtaining a non linear numerical model that included the shell shaker interaction. In particular, large amplitude vibrations were studied experimentally following a stepped sine testing procedure and it was observed that there is a strong energy transfer mechanism from low frequency axisymmetric and asymmetric modes resulting in non linear inter actions between these modes. Moreover, a saturation phenomenon regarding the vibration of the top disk was observed, associated with the violent shell vibration. The numerical simulations in Ref. [96] clarified the experimental results, and the shell model was similar to Ref. [95]. Fig. 3(a c) shows the experimental results obtained by Pellicano [96] on a circular cylindrical shell with top mass subjected to base excitation. Strong interaction between the shaker and the shell disk system can be observed from the figures. Moreover, it can be seen that the acceleration on the shell is surprisingly huge. Parametric vibrations of shells with geometric imperfections have a

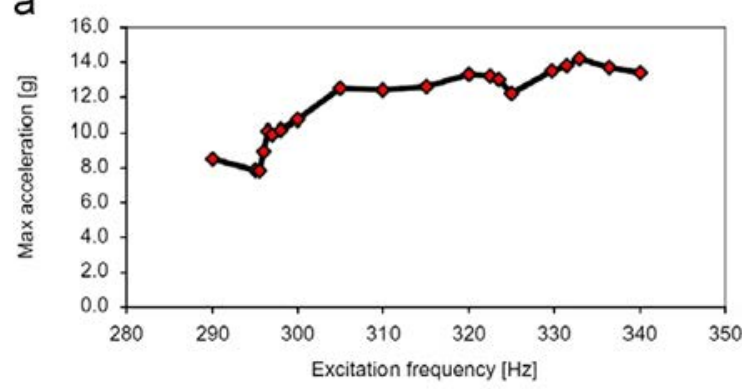

b

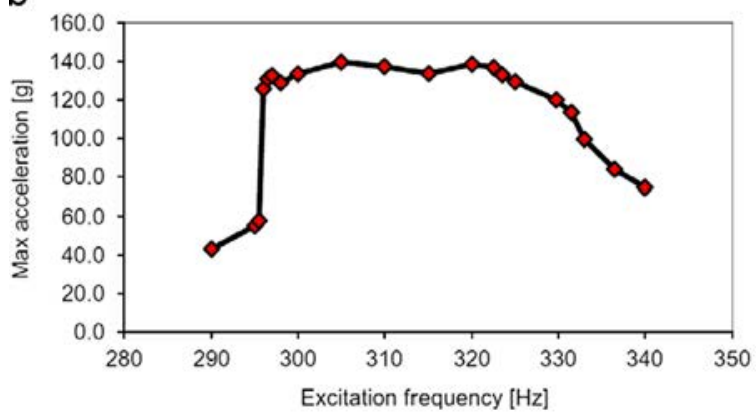

C

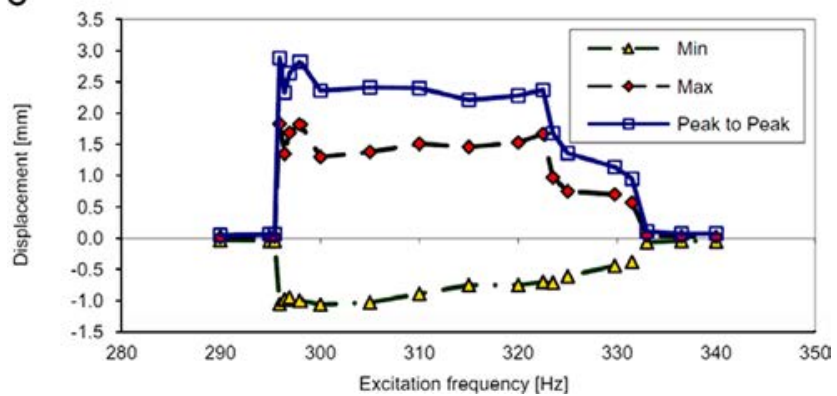

Fig. 3. Experimental results for the large-amplitude vibrations of a circular cylindrical shell with top mass subjected to base excitation. (a) Base excitation amplitude (acceleration $[g]$ ); (b) top disk amplitude (acceleration $[g]$ ); (c) maximum, minimum and the peak-to-peak of the shell response at the midspan. From Pellicano [96].

been investigated in Refs. [97 102]. Kubenko and Koval'chuk [97] analyzed the effect of initial imperfections on the stability and dynamics of circular cylindrical shells. In particular, they reviewed 193 papers in this field and studied the influence of initial axisym metric and non axisymmetric deflections on the parametric vibra tions of imperfect shells. They suggested that, although numerous studies can be found addressing the dynamic problems of circular cylindrical shells, the effect of imperfections is an important issue that should be further elaborated.

Catellani et al. [98] developed a multi mode approach based on the shell theories of Donnell and Sanders and analyzed the correlation of parametric instability with shell collapse, taking into account the effect of geometric imperfections. Pellicano and Amabli [99] extended their previous study [84] by deeply analyz ing the effect of geometric imperfection on post critical buckling, non linear vibrations and chaotic oscillations of shells subjected to combined static and periodic axial loads. To classify the non linear dynamics, they extracted the correlation dimension, calculated the Lyapunov exponents and depicted the Poincaré sections and bifurcation diagrams. Pellicano [100] also investigated the sensitivity to geometric imperfections in the dynamic stability analysis of strongly compressed circular cylindrical shells. He used the Sanders Koiter non linear theory and showed that the role of imperfections becomes important in case of shells subjected to combined axial compression 
and periodic loads since dynamic instabilities interact with the static potential well. The effect of initial geometric imperfections on the parametric vibrations of circular cylindrical shells was also investigated by Kochurov and Avramov [101]. In their analysis, Donnell's non linear shallow shell theory was used and an expansion of transverse displacement with eight degrees of freedom including six asymmetric modes (driven and companion modes), one axisymmetric mode and a constant term was utilized to obtain the equations of motion via the Galerkin approach. Then, by assuming that the frequencies corre sponding to the axisymmetric and the constant term are much higher than the asymmetric modes, the time derivatives of those modes were set to zero and a system of six second order ordinary differential equations plus two algebraic equations was solved by the method of multiple scales and a continuation algorithm. The effect of geometric imperfections on the dynamic instability of cylindrical shells carrying a top mass and subjected to base excitation was investigated by Mallon et al. [102]. Based on Donnell's shallow shell theory and considering a multi mode expansion, they obtained a semi analytical model that was verified by finite element simulations in case of linear vibration and buckling. Moreover, depending on the type of initial imperfection, they found periodic, quasi periodic and chaotic post critical behaviors for the shells under investigation.

\subsection{Fluid shell interaction}

This subsection is divided into two sections: (i) fluid structure interaction for shells containing still fluid; and (ii) fluid structure interaction for shells subjected to flowing fluid. It is worth noting that papers addressing the non linear response of shells containing still fluid also discuss the non linear vibrations of empty shells. Therefore, a reader interested in Sections 3.1 and 3.2 should also refer to Section 3.3.1.

\subsubsection{Fluid structure interaction for shells containing still fluid}

In the studies reviewed in this section, the contained fluid is assumed to be incompressible and inviscid. In fact, liquid filled shells vibrating in the low frequency range satisfy very well the incompressible assumption. Moreover, non linear effects in the dynamic pressure and in the boundary conditions at the fluid structure interface, and the shell pre stress due to fluid weight are neglected. Therefore, to determine the hydrodynamic pressure exerted by the fluid on the shell, the fluid motion has been described by the velocity potential, which satisfies the Laplace equation in cylindrical coordinates.

Non linear free vibrations of fluid filled circular cylindrical shells were investigated in Refs. [103 108]. In particular, Kubenko et al. [103] used the asymptotic Krylov Bogolyubov Mitropol'skii aver aging method to study the sub harmonic responses of shells fully filled with a perfect incompressible liquid. Circumferential traveling waves in carrier shells filled with liquid were investigated by Koval'chuk and Fillin [104]. The same authors [105] also studied the flexural vibrations of initially bent, partially filled circular cylindrical shells. Kubenko et al. [106] extended their previous work [103] and studied non linear free vibrations of fluid filled shells with internal resonance using the same averaging technique. Diverse internal resonances of simply supported and clamped shells filled with fluid were investigated by Koval'chuk and Kruk [107]. Silva et al. [108] studied the non linear free vibration response of fluid filled circular cylindrical shells following a qualitatively accu rate reduced order model obtained via the POD method. By comparing the backbone curves of empty and fluid filled shells, they showed that the internal fluid yields a stronger softening behavior. It should be noted that, in all these papers the governing equations of motion were obtained using Donnell's non linear shallow shell theory.
Amabili [56] performed a full series of comparisons between numerical and experimental results for large amplitude vibrations of fluid filled imperfect shells subjected to radial excitation. Fig. 4 ( $a$ and $b$ ) shows the comparison between the experimental and numerical frequency response curves of a water filled shell with the number of circumferential waves equal to 10 . The presence of one to one internal resonance between driven and companion modes appear in both theoretical and experimental curves. Amabili [57] compared the responses of different non linear theories in the case of fluid filled shells and showed that, although Donnell's non linear shallow shell theory is the least accurate theory for empty shells, it gives sufficient accurate results for water filled shells. This was because the in plane inertia, which is

a

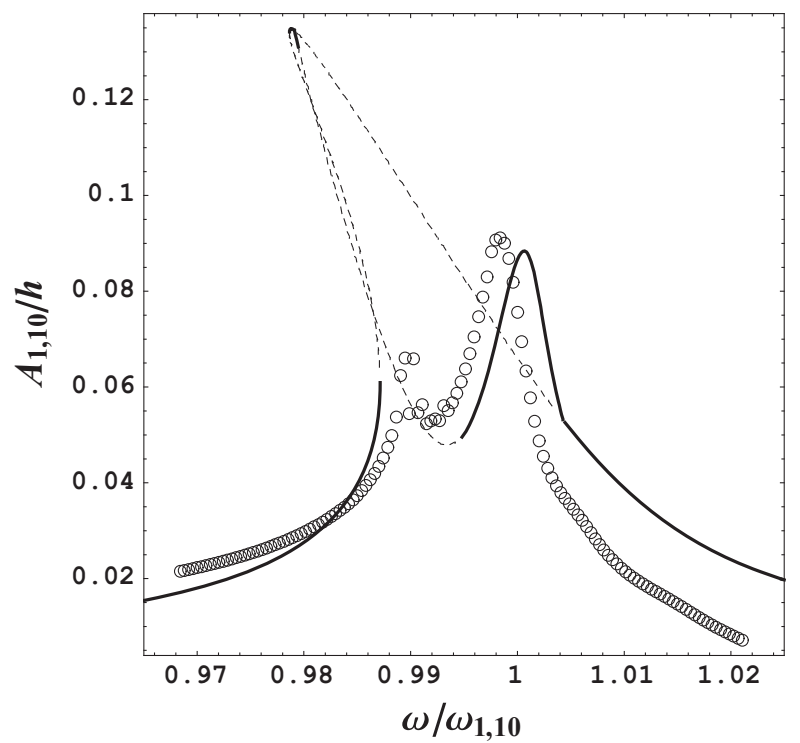

b

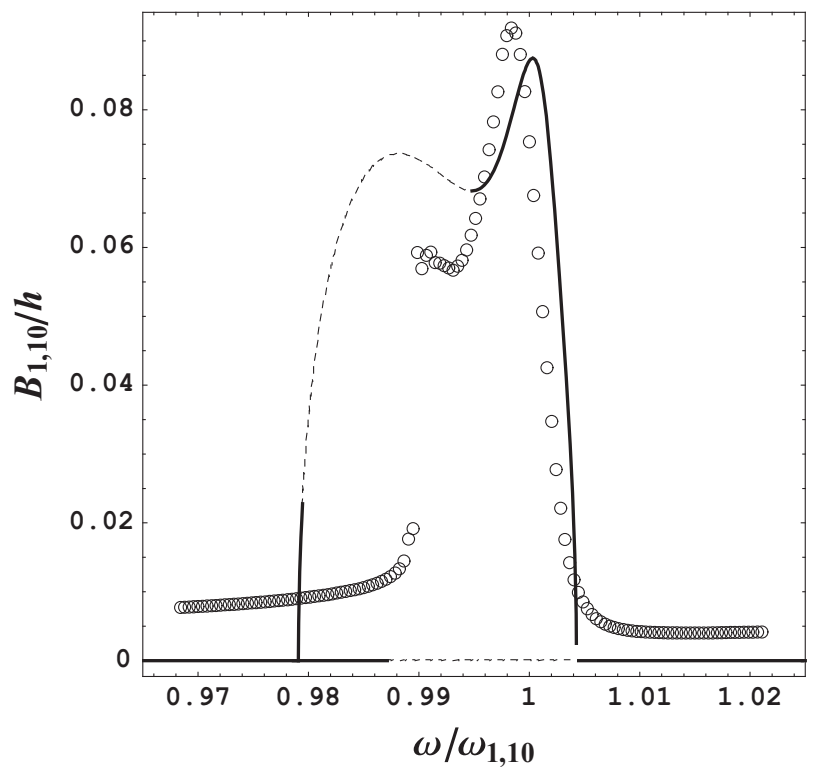

Fig. 4. Comparison between experimental and theoretical frequency-response curves for a water-filled shell ( $n 10, m 1$ ); force $3 \mathrm{~N}$ and is applied in the radial direction, $\omega$ is the excitation frequency. Experimental data; — stable solution; - - - unstable solutions. (a) Amplitude of the driven mode/ $h$; (b) amplitude of the companion mode/ $h$. $h$ Denotes the thickness of the shell. From Amabili [56]. 
neglected in Donnell's shallow shell theory, is less important for a water filled shell that has a large radial inertia owing to the presence of internal liquid.

The chaotic vibrations of partially filled shells were investigated by Koval'chuk and Kruk [109] using a model that had only two degrees of freedom. Karagiozis et al. [110] developed two numer ical models based on Donnell's non linear theory with and with out in plane inertia to study non linear vibrations of clamped fluid filled shells. In a series of papers Koval'chuk and Kruk [111,112], Koval'chuk et al. [113] and Kubenko et al. [114] discussed the problem of non linear forced vibrations of completely filled simply supported circular cylindrical shells using Donnell's non linear shallow shell theory and the Krylov Bogolyubov Mitro pol'skii averaging technique. Particularly, in Refs. [111,112,114] a three degree of freedom model was used that included the driven and companion modes plus a quartic sine term as a function of longitudinal coordinate to describe the axisymmetric contraction of the shell, whereas in Ref. [113] a five degree of freedom model that contained four asymmetric modes was considered to study the non linear interaction of different flexural modes. The quartic sine term was used in the expansion of the transverse displace ment instead of the well known squared sine, to satisfy the moment resultant at the edges of the shell exactly.

Reduced order models based on POD and the non linear normal modes method, for studying large amplitude vibrations of fluid filled shells subjected to radial harmonic excitation, have been obtained in Refs. [108,115 118]. In particular, Amabili et al. [115,116] compared the Galerkin and POD models of a water filled cylindrical shell for moderate and large amplitude vibrations. In Ref. [115] it was shown that the POD technique allows a drastic reduction in the computa tional effort. Moreover, it was illustrated that models with only three degrees of freedom based on POD have the same level of accuracy as models built by the Galerkin technique with sixteen degrees of freedom. In Ref. [116], it was found that more proper orthogonal modes are necessary to reach energy convergence for chaotic dynamics and it is important to extract the modes using a time series of complex responses (quasi periodic or chaotic). Touzé and Amabili [117] used the non linear normal modes method and built a reduced order model with only two degrees of freedom that showed accurate results for vibration amplitudes up to twice the thickness of the water filled shell. The detailed comparison between POD and non linear normal modes method in studying non linear vibrations of fluid filled shells was given by Amabili and Touzé [118]. Silva et al. [108] combined a perturbation technique and the POD method and assessed the quality of the proposed method by studying the convergence of frequency amplitude curves, bifurcation diagrams and time responses.

Parametric vibrations of shells conveying fluid were investigated in Refs. $[84,88,99,108,119]$. Pellicano and Amabili [84] showed that compared to empty shells, the presence of liquid changes the dynamic stability bounds interestingly. Gonçalves et al. [88] discussed the significance of the transient response, in studying the dynamic buckling of fluid filled shells. Pellicano and Amabili [99] confirmed that the presence of fluid provides a safety effect on the onset of instability that occurs via period doubling bifurcation. In the same paper, they discussed the chaotic response of fluid filled imperfect shells under periodic axial loads. Parametric instability boundaries and chaotic vibrations of fluid filled cylindrical shells were also studied by Silva et al. [108]. The same authors [119] also discussed the dynamic instability and snap through buckling of partially filled shells.

\subsubsection{Fluid structure interaction for shells subjected to flowing fluid}

Systematic research on the non linear dynamics of shells conveying fluid was conducted by Païdoussis and it is synthesized in his monograph [120].
Theory and experiments for the dynamic stability of circular cylindrical shells subjected to incompressible subsonic liquid and air flow have been reported by Karagiozis et al. [121 125]. In the theoretical part of these studies, the shell was assumed to be in contact with inviscid fluid, and the fluid structure interaction was described by the potential flow theory. Experiments on non linear dynamics of clamped shells subjected to axial flow were described in Ref. [121] and its visual experimental evidence was provided in Ref. [122]. A subcritical non linear softening behavior was reported for shells subjected to internal and external flows. Three different types of experimental tests were carried out on fully clamped shells in Ref. [123], which included experiments on (i) elastomer shells in annular airflow; (ii) elastomer shells with internal airflow; and (iii) aluminum or plastic cylindrical shells with internal water flow. It was found that the interaction between the shell and the fully developed flow gives rise to instabilities in the form of static or dynamic divergence at sufficiently high flow velocities. In Ref. [124], the effect of varying the thickness to radius and length to radius ratios of the shell on non linear stability was discussed and it was found that an increase of the circumferential wave number, for shells with the same thickness to radius ratio, enhances the subcritical behavior of the shell. Karagiozis et al. [125] elaborated the experiments performed in Ref. [123] and compared the experimental results with numerical ones obtained for simply supported and fully clamped shells in details. Shells with internal and annular fluid flows were studied and it was found that the system loses stability by static, strongly subcritical divergence. In order to obtain the numerical results, Donnell's non linear shell theory was used and the Galerkin approach was employed to obtain the equations of motion, which were solved using a pseudo arc length continuation and collocation method and Gear's backward differentiation formula. For fully clamped shells, models having seven (4 asymmetric modes, 3 axisymmetric modes), eight (4 asymmetric modes, 4 axisymmetric modes) and eleven (8 asymmetric modes, 3 axisymmetric modes) degrees of freedom were considered, whereas for the simply supported shell the numerical model had eighteen degrees of freedom (12 asymmetric modes, 6 axisymmetric modes).

The effect of imperfections on the non linear stability of shells containing fluid flow was investigated by Amabili et al. [126] using a refined model. A Lagrangian approach based on (i) Donnell's theory retaining in plane inertia and (ii) the Sanders Koiter theory was utilized retaining 51 degrees of freedom in the discretization and differently from previous works, the effect of fluid viscosity was taken into account by using the time averaged Navier Stokes equa tions. Non classical boundary conditions were used to simulate the conditions of experimental tests in a water tunnel and satisfactory comparisons of numerical and experimental results were conducted. It was shown that asymmetric geometric imperfections with the same number of circumferential waves as the mode associated with instability play a significant role, transforming the pitchfork bifurca tion at divergence to a folding (saddle node) bifurcation. Good agreement was shown with the available experimental results for divergence of aluminum shells conveying water. Moreover, it was found that axisymmetric and asymmetric imperfections with a number of circumferential waves that is not a multiple of the number of waves at instability play a small role.

The combined effect of geometric imperfections and fluid flow on the non linear vibrations and stability of shells was investi gated by del Prado et al. [127].The behavior of the thin walled shell was modeled by Donnell's non linear shallow shell theory and the shell was assumed to be subjected to a static uniform compressive axial pre load plus a harmonic axial load. A low dimensional model was obtained using the Galerkin method and the numerical solutions were found using a Runge Kutta scheme. It was shown that the parametric instability regions, bifurcations and basins of attraction are affected by the initial geometric imperfection and flow velocity. 
The effect of fluid viscosity was also retained by Karagiozis et al. [128] in studying the non linear vibrations of harmonically excited circular cylindrical shells conveying water flow. A detailed analysis was carried out using a pseudo arc length continuation method at different flow velocities and for (i) fixed excitation amplitude and variable excitation frequency and (ii) fixed excitation frequency and variable excitation amplitude. Moreover, in order to classify the system dynamics, direct time integration of equations of motion was performed to obtain bifurcation diagrams of Poincaré maps, the maximum Lyapunov exponent and the Lyapunov dimension. Periodic, quasi periodic, sub harmonic and chaotic responses were detected, depending on the flow velocity and amplitude of the harmonic excitation. Fig. 5( $a$ and b) shows the non linear forced response of the driven and companion modes of a simply supported shell subjected to external harmonic excitation and different flow velo cities. It is interesting to see that the softening behavior is enhanced by increasing the flow velocity.

By neglecting the effect of fluid viscosity and considering the potential flow model, non linear forced vibrations and stability of

a

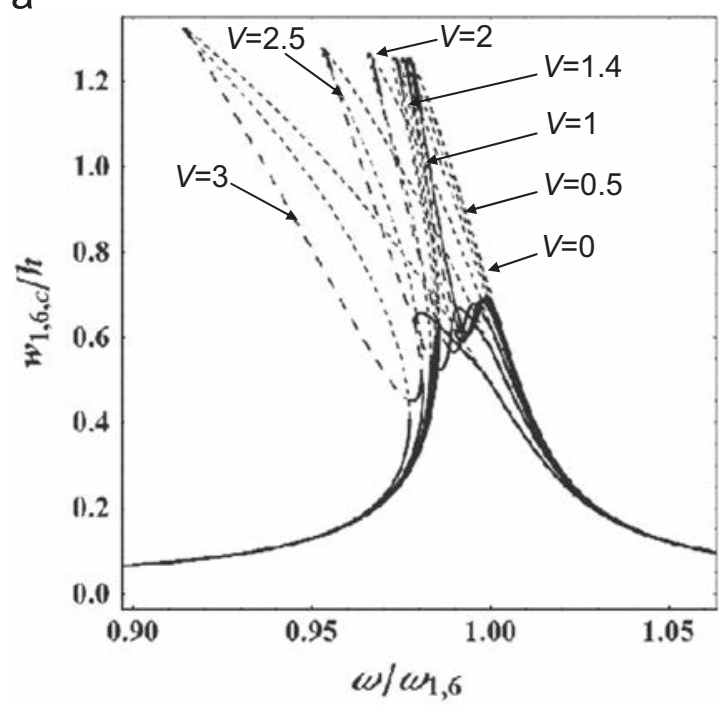

b

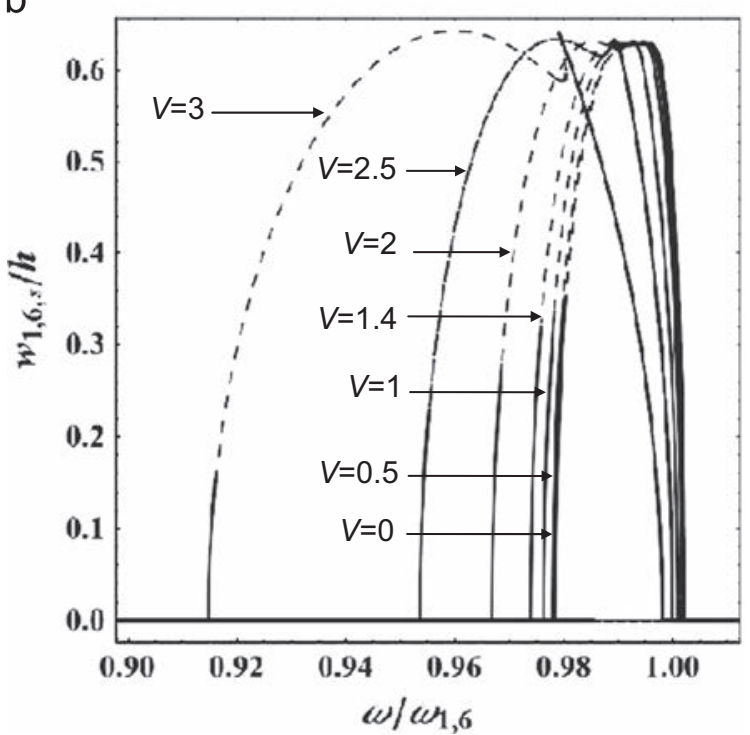

Fig. 5. Effect of flow velocity on non-linear frequency-response curves. Force $0.0165 \mathrm{~N}$. (a) Amplitude of the driven mode $/ h$; (b) amplitude of the companion mode $/ h$. $h$ Denotes the thickness of the shell and $V$ is the flow velocity. From Karagiozis et al. [128]. shells interacting with fluid flow were investigated in Refs. [129 132]. Koval'chuk [129] used Donnell's non linear theory together with the Galerkin approach and the Krylov Bogolyubov Mitropol'skii aver aging technique to study the non linear vibrations of the shell, neglecting the effect of axisymmetric modes. The same theory and solution methodology was used by Koval'chuk and Kruk [130]. However, in their analysis, the numerical model had six degrees of freedom, which included four asymmetric modes plus two axisym metric modes. The axisymmetric modes were described as quartic sine terms. Kubenko et al. [131] extended the previous works of Refs. $[129,130]$ by showing the mathematical procedure for the Krylov Bogolyubov Mitropol'skii method in studying multi mode non linear free, forced and parametrically excited vibrations of shells in contact with flowing fluid. Kubenko et al. [132] also studied the vibrations of cylindrical shells interacting with a fluid flow and subjected to external periodic pressure with slowly varying frequency.

Non linear dynamics of cantilevered circular cylindrical shells subjected to flowing fluid was investigated by Paak et al. [133]. The non linear model of the shell was based on the Flügge theory retaining non linear terms due to mid surface stretching, and the fluid model was based on the potential flow theory. The unsteady interaction and asymptotic dynamics of a viscous fluid with an elastic shell were also examined by Chueshov and Ryzhkova [134] using the linearized Navier Stokes equations and Donnell's non linear shallow shell theory.

Non linear vibration of circular cylindrical shells in supersonic flow and non linear flutter instability of shells is an important subject. Little publications can be found in the last decade on non linear studies dealing with isotropic shells, whereas publications concerning linear studies or non linear analysis of non isotropic shells are wider (see Section 5.3). The only publications available since 2003 are those by Kurilov and Mikhlin [135] (even if the spelling of both name and surname is different, the first author is the same author of Refs. [73,74] ) and Jansen [136]. Donnell's non linear shallow shell theory was used in both works. However, in Ref. [135] a pseudo arc length continuation scheme was used to solve the equations of motion, whereas in Ref. [136] the non linear response was obtained following a semi analytic perturbation method. Earlier investigations in this field were carried out by Amabili and Pellicano [137,138] and new results based on this approach were published in Ref. [5].

\subsection{Forced vibrations of shells subjected to other types of loading conditions}

The non linear response of circular cylindrical shells subjected to dynamic transient loading was investigated by Sansour [139] using the finite element method. A time integration scheme was proposed and formulated for a shell with seven parameters and with arbitrary geometric and material non linearities, which conserved energy, momentum and angular momentum.

Non linear vibrations of isotropic circular cylindrical shells in thermal environments were investigated in Refs. [78,79,140,141]. Awrejcewicz and Krysko [140] proposed an approach based on finite difference and a relaxation method to solve the coupled thermoelastic problem of a flexible shallow shell. Shu et al. [141] studied the coupled thermoelastic vibrations of circular cylindrical shells in large deflection. In their analysis, the non linear terms were only retained in the strain displacement relation in the longitudinal direction, and a single mode approximation was used to study the effect of temperature on the vibration amplitude.

Dynamic buckling of imperfect shells under short term impul sive loads was reviewed by Kubenko and Koval'chuk [97]. Moreover, large amplitude vibrations of cylindrical shells subjected to non stationary loads (e.g. impulse) were investigated in Refs. [142 148]. Shul'ga and Bogdanov [142] developed a numerical method based on 
the integro differential method of constructing difference schemes to find the response of conical shells with different taper angles subjected to an impulsive load. The same authors [143] proposed a numerical scheme based on the finite difference method to study the dynamic stress strain state of reinforced circular cylindrical shells subjected to different initial deflections. Vibrations of discretely ribbed, reinforced shells were also investigated by Meish and Kairov [144] based on the Timoshenko shell theory and using a finite difference scheme. In a series of papers, Lugovoi et al. [145 147] used the same theory and extended the work of Ref. [144] by solving the problem via a new technique based on the Richardson extrapolation method in [145], studying the dynamics of compound cylindrical shells with spherical caps in [146], and investigating the dynamic behavior of cylindrical shells in an elastic medium in [147]. Moreover, the stability of vibration mode transformation between extensional and flexural modes, and two to one internal resonance in thin walled circular cylindrical shells subjected to large initial radial velocity or impulse was investigated by Shi et al. [148].

Large amplitude vibrations of shells subjected to electrical loads were investigated in Refs. [149 152]. In particular, using the three dimensional equations of non linear electroelasticity, Yang et al [149] derived a single degree of freedom model, which was solved by the method of harmonic balance to obtain the non linear frequency amplitude behavior of the electric current near resonance. Karnaukhov and Tkachenko [150] studied the vibrations of an infinitely long thin cylindrical shell made of a non linear elastic piezoceramic material subjected to periodic electric force. They used the method of harmonic balance to plot frequency response curves and time responses, for different levels of electric loading. The non linear vibration response of a thick walled spherical shell subjected to mechanical pressure and electric field was investigated by Yong et al. [151]. They assumed that the dielectric elastomer is isotropic and neo Hookean hyperelastic, and found the critical voltage for various loading conditions. Moreover, they found that the spherical shell may undergo quasi periodic motion while it is subjected to sinusoidal electrical load. The same authors [152] also studied the non linear vibrations of dielectric elastomer circular cylindrical shells modeled by neo Hookean material, when subjected to a periodic voltage. They discussed the influence of thickness and boundary conditions on the critical voltage and stated that by applying a voltage greater than the critical value, the shell will be destroyed. Moreover, to obtain periodic solutions, they used a shooting method.

Dong et al. [153] studied non linear modal coupling and strain growth phenomenon in spherical shells subjected to blast loading. Using the LS DYNA finite element software, they explained that the strain growth in the non axisymmetric response of closed spherical shells is due to the non linear coupling between the radial breathing mode of the shell and its composite modes.

Non linear vibrations and dynamic stability of viscoelastic circular cylindrical shells subjected to a dynamic load (i.e. ramp) irregularly distributed along the surface of the shell were investigated in a series of papers by Eshmatov [154 156]. In all these papers, the equations of motion were derived based on the Timoshenko theory and taking into account shear deformation and rotary inertia. Moreover, by assuming simply supported boundary conditions and by applying the Galerkin approach, the equations of motion were reduced to a set of non linear integro differential equations, which were solved via a quadrature technique.

\section{Non-linear vibrations of isotropic panels}

This subsection is divided into two sections: (i) cylindrical and doubly curved panels; (ii) spherical caps, and panels of other geometries.

\subsection{Cylindrical and doubly curved panels}

Non linear vibrations of cylindrical and doubly curved panels (shallow shells) with rectangular base were investigated in depth by Amabili [157 161]. Experiments on large amplitude vibrations of circular cylindrical panels were performed in Ref. [157]. The experi mental boundary conditions that were considered allowed zero axial, radial and circumferential displacements on the curved edges and zero axial and radial displacements on the straight edges. A stepped sine testing technique was performed in the frequency neighborhood of the first three natural frequencies of the tested cylindrical panel and frequency response curves were obtained that showed strong soft ening behavior. Amabili $[158,159]$ extended his work by addressing the non linear vibrations and internal resonances of simply supported cylindrical [158] and doubly curved panels [159], numerically. The shell theories of Donnell and Novozhilov were used and a Lagrangian approach was utilized to obtain the equations of motion. Moreover, the bifurcation analysis was performed by using a pseudo arc length continuation and collocation scheme and in order to check the convergence of the solution, different multi dimensional models were considered. It was found that differently from closed circular cylind rical shells that show weak softening behavior, shallow cylindrical and spherical shells show a softening type non linearity that may turn to hardening for large amplitude vibrations. Moreover, it was shown that hyperbolic paraboloidal shells (cases with negative curvature ratios) show hardening type non linearity. Fig. 6 shows the trend of non linearity for different types of shallow shells. Comparisons between experimental results of circular cylindrical panels and simulations taking into account the geometric imperfections were performed by Amabili [160]. Donnell's non linear theory was used and the equations of motion were obtained via a Lagrangian approach. For experiments, two thin cylindrical panels were tested for several excitation ampli tudes to characterize the non linearity. The dimensions of the two panels were chosen in order to have fundamental modes with one and two circumferential half waves, respectively. Amabili [161] also dis cussed the effect of different boundary conditions on the trend of non linearity of circular cylindrical panels.

Finite element studies for large amplitude vibrations of cylindrical panels can be found in Refs. [162 168]. Sansour et al. [162] proposed

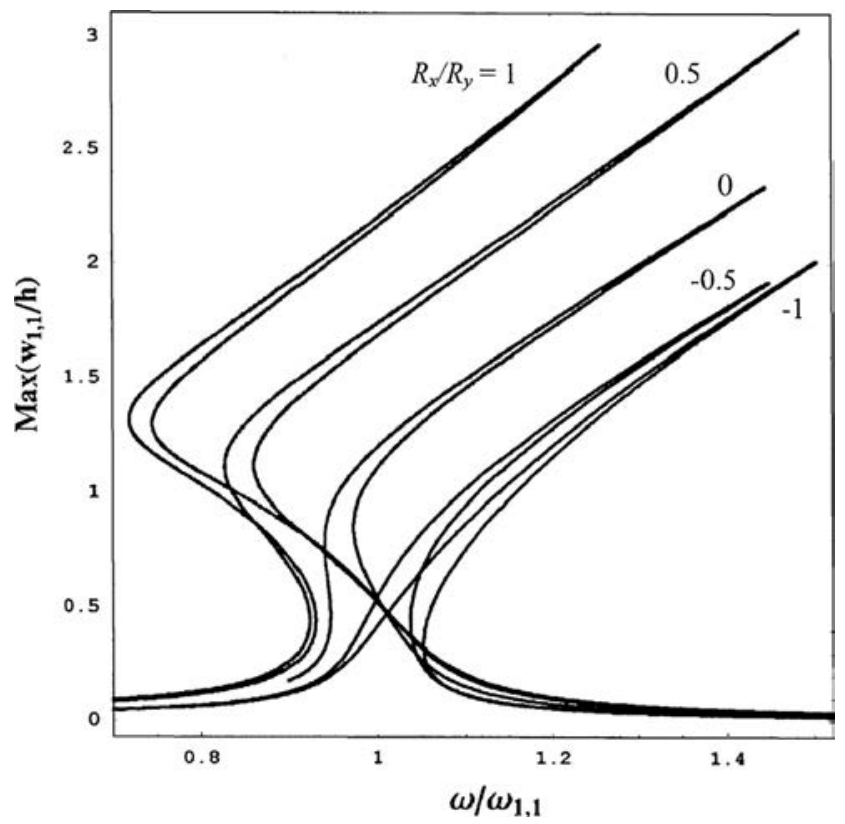

Fig. 6. Effect of curvature ratio $\left(R_{x} / R_{y}\right)$ on the trend of non-linearity in doubly curved panels. $\omega_{1,1}$ Is the fundamental frequency, $\omega$ is the excitation frequency and $w_{1,1}$ is the fundamental generalized coordinate. From Amabili [159]. 
a post processed Galerkin finite element based on hierarchical inter polations to study the non linear response of cylindrical and spherical panels subjected to transient dynamic loads. Ribeiro [163] used first order shear deformation theory and $p$ version finite element with hierarchic basis functions to study non linear free vibrations of moderately thick isotropic doubly curved shallow shells. Shells with different boundary conditions, curvature ratios and thicknesses were studied and it was found that in order to reach accurate results, the $p$ version finite element requires fewer degrees of freedom than the $h$ version finite element method. de Faria [164] assessed the dynamic response of cylindrical panels by studying the effect of moving mass, moving force and load velocity. The equations of motion that were obtained by the finite element method in [164] were non linear only in the velocity of the moving mass, and a perturbation technique was proposed to convert the non linear equations to a series of linear ordinary differential equations. Finite element formulations for large amplitude vibrations of isotropic shallow panels were suggested by Przekop et al. [165]. Non linear first order shear deformation theory was used and it was found that even for moderate deflections, higher modes contribution can be quite large. Ribeiro $[166,167]$ used the same theory and solution methodology of Ref. [163] to illustrate the internal resonances that occur between non linear modes and to study the non linear forced vibrations of shallow cylindrical panels, respectively. In particular, in Ref. [166] the harmonic balance method with a constant term and three harmonics was used to transform the equations of motion into frequency domain equations, which were then solved using a continuation method to obtain the backbone curves, whereas in Ref. [167] a shooting method in conjunction with the Newton method was utilized to solve the equations of motion and to obtain the non linear frequency response curves. Similar finite element formulations were presented by Ribeiro et al. [168] to study non linear vibrations of deep cylindrical panels.

Touzé et al. [63] used non linear normal modes method to study geometrically non linear forced vibrations of simply sup ported hyperbolic paraboloidal shells, and cylindrical panels with simply supported, in plane free edges. They showed that a reduc tion to a single non linear normal mode is possible, showing very accurate results for moderate amplitude vibrations. Alijani et al. [169] discussed the accuracy of the method of multiple scales for non linear vibrations of doubly curved panels. Donnell's non linear shallow shell theory was used and the Galerkin method was applied to obtain the governing equations of motion. In order to perform a perturbation analysis and to study the primary resonance response, a quadratic relation was assumed between the excitation frequency and the fundamental frequency of the panel. It was shown that, although in case of hardening non linearity (shallow shells with large radii of curvature) the results resemble those found by numerical integration or continuation methods, in case of softening non linearity (e.g. spherical panels) the solution breaks down as the amplitude becomes greater than the thickness.

Non linear vibrations of shallow shells having a complex shape have been investigated in Refs. [170 175] based on the R functions mesh free technique. The $\mathrm{R}$ functions method is a powerful tool to obtain discretization of two dimensional and three dimensional domains of complex shape, and can be applied to moving boundaries. Kurpa et al. [170] obtained a single degree of freedom model for different sets of boundary conditions and presented the backbone curves. The results obtained by the $\mathrm{R}$ functions method were in good agreement with the results previously obtained by Amabili $[158,159]$ for simply supported panels and experimental results performed on a cylindrical panel with complex boundary conditions. In particular, the complex panel had symmetrical squared grooves on the straight edges of the panel. Breslavsky and Avramov [171] studied a similar problem by using the $\mathrm{R}$ functions method together with a combination of non linear normal modes and multiple scales method to obtain the backbone curves of a complex cylindrical panel with symmetrical squared grooves on the curved edges. The same problem was also studied by Breslavsky and Avramov [172] using a combination of $\mathrm{R}$ functions and the harmonic balance method. The non linear vibrations of complex base shallow shells submerged in a fluid were investigated by Breslavsky et al. $[173,174]$. The interaction of the shell with the fluid was described by a hyper singular integral equation, which was solved by the boundary element method, and the method of normal modes was used to study the large amplitude vibrations. Pilgun and Amabili [175] performed a novel two step Lagrangian approach to study non linear forced vibrations of cylindrical panels with complex shape and clamped edges. A linear analysis was conducted in the first step, to identify natural frequencies and corresponding natural modes of the panel using Chebyshev polynomials as trial functions. Then, the natural modes obtained from the first step were used as the basis for non linear displacements. Both Donnell and Sanders Koiter the ories were used and the bifurcation analysis was carried out using a pseudo arc length continuation and collocation technique.

Chaotic vibrations and complex non linear dynamics of panels have received considerable research attention in the literature. Sansour et al. [176] constructed an energy momentum integration scheme in the framework of the finite element method to study the chaotic motion of panels. Nagai et al. [177] studied the effect of concentrated mass on the chaotic vibrations of shallow cylindrical panels. They used Donnell's non linear theory and the Galerkin approach to obtain the governing equations of motion. The chaotic responses of the shell were examined by Poincaré maps, Lyapunov exponents and Lyapunov dimension following direct time integra tion of the equations of motion using a Runge Kutta method. It was illustrated that, once the concentrated mass is increased, the maximum Lyapunov exponent decreases gradually. Amabili $[159,161]$ used Gear's backward differentiation formula (BDF) to perform bifurcation analysis on multi modal numerical models and to obtain the bifurcation diagrams, Poincaré maps, Lyapunov exponents and Lyapunov dimension of a spherical and a cylindrical panel, respectively. He found that panels exhibit interesting non linear phenomena such as sub harmonic response, period doubling bifurcation, quasi periodic and chaotic behaviors when they are subjected to large amplitude excitations. Krysko et al. [178] used the Ritz method in conjunction with the fourth order Runge Kutta time integration technique to investigate the chaotic response of spherical panels subjected to sign changing transversal harmonic load. Awrejcewicz et al. [179] studied the transition from regular to chaotic motion in spherical panels with non homogenous thick ness. The chaotic vibrations of shallow panels were experimentally investigated by Nagai et al. [180]. The tested panel was simply supported on all edges, and was subjected to gravity and periodic lateral excitation. Two types of chaotic responses were identified that were bifurcated from a sub harmonic resonance response of half order and an ultra sub harmonic resonance response of two third order corresponding to the fundamental mode of vibration. Wang et al. [181] studied the chaotic response of cylindrical reticulated panels by considering a single degree of freedom model and using the Melinkov method. Awrejcewicz et al. [182] studied the chaotic dynamics of cylindrical panels with infinite length subjected to harmonic loads. They found classical routes to chaos including Feigenbaum and Ruell Takens Newhouse scenarios. Mar uyama et al. [183] extended the work of Nagai et al. [180], and provided detailed numerical and experimental results for modal interactions of doubly curved shallow panels. The contribution of modes of vibration in the chaotic response was discussed in Ref. [183]. Additional experimental and numerical analyses were per formed by Nagai et al. [184] to identify chaotic regions of cylindrical panels with a combination of clamped and simply supported boundary conditions and subjected to lateral periodic acceleration. Krysko et al. [81] observed Feigenbaum scenario associated with 
non smooth stability loss for cylindrical panels subjected to uni formly distributed transversal harmonic load. In the same paper, the Ruelle Takens Newhouse route to chaos was found for spherical panels.

Non linear vibrations of viscoelastic panels were investigated in Refs. [185 188]. Khudayarov and Bandurin [185] proposed a numerical algorithm to solve the integro differential equations of viscoelastic panels in contact with gas flow. Eshmatov and Khodzhaev [186,187] studied the effect of concentrated masses on the non linear vibrations and dynamic stability of viscoelastic cylindrical panels, respectively. The Koltunov Rzhanitsyn singular kernel with three rheological parameters was used for the viscoe lastic model and it was observed that an increase in the concen trated mass leads to a sharp drop in the frequency and amplitude of the panel. The effect of damping on the non stationary vibra tions of a viscoelastic simply supported cylindrical panel with distributed piezoelectric sensors and actuators was investigated by Karnaukhov and Tkachenko [188]. An integral viscoelastic model was used to describe the viscoelastic behavior of the material, and the effect of viscosity and geometric non linearity on the resonant vibrations of the cylindrical panel was discussed.

\subsection{Spherical caps and panels of other geometries}

Spherical caps are segments of spherical shells with circular base described in spherical coordinates. Non linear dynamics of shallow spherical caps subjected to radial dynamic edge loading (in the form of step loading) was investigated by Odeh [189]. He found that snap through buckling is possible under peripheral dynamic loading condition and the amount of snapping is con siderable when the cap has an opening around the apex.

In a series of papers, Thomas et al. [190], Touzé and Thomas [191], Thomas et al. [192] and Camier et al. [193] investigated in depth the non linear vibrations of free edge spherical caps. In particular, Thomas et al. [190] used Donnell's shallow shell theory and a set of eigen modes obtained from the associated linear problem to calculate all coefficients of the non linear quadratic and cubic terms that appear in the governing equations of motion and discussed the non linear modal interaction rules. Moreover, by deriving a three degree of freedom model and using the method of multiple scales, a specific mode coupling due to $1: 1: 2$ internal resonance between two companion modes and an axisymmetric mode was studied. Touzé and Thomas [191] used the non linear normal modes method to study the effect of geometry on the trend of non linearity. They found that, similar to closed circular cylind rical shells, axisymmetric modes play an important role in the non linear behavior of purely asymmetric modes. Moreover, it was observed that the behavior of asymmetric modes is generally hardening, and it may become softening for certain cases after a 2:1 internal resonance between an asymmetric and an axisym metric mode. It was also shown that, the behavior of axisymmetric modes may change from hardening to softening for small values of aspect ratios, since the eigen frequencies of the axisymmetric modes depend greatly on the curvature of the spherical cap. Thomas et al. [192] performed experimental tests to validate the occurrence of 1:1:2 internal resonance in free edge spherical caps. The particular internal resonance was observed between the first axisymmetric mode and the sixth asymmetric modes. A coil magnet non contact exciter was used to excite the shell and three transducers, two accelerometers and a laser Vibrometer were used to measure the time evolution of the three involved modes. Particularly, the shell was excited at the center so that only the axisymmetric mode was directly excited and the laser Vibrometer beam was pointing at the center to measure the time response of the axisymmetric mode only. Experimental frequency response curves were obtained for different force levels and quantitative differences were found between the experimental and theoretical results owing to geometric imperfections that were not included in the numerical model. In order to find consistent experimental and numerical results, Camier et al. [193] improved the previous theoretical models of Refs. [190 192] by taking into account the effect of geometric imperfections. They found that, although good agreement can be seen between the linear experimental and numerical results, there are still discrepancies between the non linear results. In fact, it was observed that the theory over predicts the instability regions, even though it correctly predicts the trend of non linearity. A very possible reason of the discrepancy was mentioned to be the thickness variation or inhomogeneous material property of the tested shell, which was still being neglected in the numerical model.

Non linear vibrations of corrugated spherical shallow panels were investigated by Yuan and Liu [194] and Yuan [195]. Green's function method was used to reduce the problem to a single degree of freedom model with quadratic and cubic non linearities and the solution was found using the Galerkin method. Wang and Song [196] studied non linear free vibrations of uniformly heated bimetallic shallow spherical caps. The Kantorovich averaging method was used to convert the governing partial differential equations to ordinary differential equations and a shooting method was used to obtain the numerical solutions. It was found that the non linear fundamental frequency decreases with tem perature increase and increases once the shell height is increased.

Chaotic vibrations of spherical caps can be found in Refs. [197 199]. Soliman and Gonçalves [197] studied the complex axisym metric dynamics of clamped spherical caps under large harmonic loads. They showed that steady state instabilities including jumps to resonance, sub harmonic response, period doubling bifurcation and cascades of period doubling bifurcations to chaos may occur by varying different parameters of the system. Moreover, they found that a sudden pulse of excitation may lead to the transient failure of the shell. Krysko et al. [198] studied the complex dynamics of spherical caps subjected to transverse distributed sign changeable load and analyzed the scenarios of transition from regular to chaotic motions.

The non linear theory of spherical caps was also used for modeling percussive instruments and loudspeakers. Chaigne et al. [199] discussed the nature of non linear coupling and chaotic response that leads to particular sounds of gongs and cymbals, by calculating the Lyapunov exponents. Bilbao [200] used the non linear shell theory and a finite difference scheme to discuss the sound synthesized by gongs due to large amplitude vibrations. Quaegebeur et al. [201] used a time domain formulation to predict the non linear sound radiation pattern of a prototype loudspeaker that was modeled as a truncated cone with a spherical cap, during large amplitude vibrations.

Non linear free and forced vibrations of shallow conical panels can be rarely found in the literature. The non linear behavior of conical panels under the combined action of peripheral moments and transverse loads was studied by Zhao et al. [202] based on a perturbation method. Thermoelastically coupled non linear axi symmetric vibrations of thin conical panels were investigated by Wang and Dai [203] by applying the Galerkin technique and by assuming similar shape functions for the fundamental mode, thermal bending moment and thermal in plane force resultant. In Ref. [203], a three degree of freedom model was obtained that was solved using the method of multiple scales. A perturbation method was also used in Ref. [204] to obtain the non linear natural frequencies of variable thickness conical shells.

Chaotic vibrations of shallow conical panels can be found in Refs. $[81,178,179,205]$. Krysko et al. $[81,178]$ found the Feigenbaum scenario for conical panels subjected to large harmonic excitations. Awrejcewicz et al. [179] discussed the routes to chaos of conical 
panels with non homogenous thickness. Wang et al. [205] used the Melinkov method to discuss the chaotic response of a conical lattice shell modeled as a single degree of freedom system.

Non linear forced vibrations of discretely reinforced ellipsoidal panels under impulsive loads were investigated by Meish [206]. Non linear first order shear deformation theory was used and a finite difference scheme was utilized to obtain the numerical solutions. The same theory and solution methodology was used by Meish and Maiborodina [207,208] to study non axisymmetric vibrations of ellipsoidal [207] and reinforced ellipsoidal panels [208] subjected to non stationary loads.

\section{Non-linear vibrations of composite shells}

This section is divided into three subsections: (i) closed composite shells; (ii) composite panels; (iii) fluid structure inter action in composite shells. It must be noted that papers dealing with vibrations of anisotropic shells generally treat vibrations of single layer isotropic shells as specific cases. Therefore, a reader interested in Section 3 should also refer to Section 5.

\subsection{Closed composite shells}

Toorani [209] and Toorani and Lakis [210] studied non linear free vibrations of composite circular cylindrical shells based on a finite element method and a shear deformation theory. In a series of papers, Jansen [211 215] used Donnell's non linear shell theory and a semi analytic method for studying non linear free and forced vibrations of orthotropic and composite shells. In particular, Jansen [211] presented two types of solutions with different levels of accuracy and complexity. In the first type, which he denoted as "simplified analysis", a number of asymmetric and axisymmetric modes are used to build reduced order models via the Galerkin approach. The obtained reduced order models are then solved either by an averaging technique or by numerical time integration. In the second type of analysis, referred to as the "extended analysis", the boundary conditions of the shell are satisfied exactly and the Fourier decomposition method is used to eliminate the dependence of the solution on the circumferential coordinate. Finally, the resulting boundary value problem for ordinary differ ential equations is solved numerically by means of a parallel shooting method while a perturbation method is used to assess the influence of large amplitude vibrations. Jansen [211] used these methods to obtain non linear frequency response curves of isotropic and orthotropic shells and to discuss the reasons of discrepancies between the results presented by different research ers. Jansen [212,213] used both simplified and extended methods to study the effect of geometric imperfections and static loading on the large amplitude vibrations of composite shells. He found that, for unloaded shells, asymmetric imperfections mostly influ ence the linear vibration behavior, while their effect on large amplitude vibration is small. Moreover, he showed that certain axisymmetric imperfections reduce linear frequencies and result in a less softening non linearity, whereas static compressive axial loads yield stronger softening behavior. In Ref. [214], the so called extended analysis was elaborated and non linear vibrations of laminated shells were studied. Jansen [215] also used the semi analytic method based on the perturbation expansion (extended analysis) to study the effect of different boundary conditions on the non linear vibrations and flutter of composite shells. These works were then extended by Rahman et al. [216] who proposed a finite element perturbation method for studying non linear free vibrations of composite circular cylindrical shells. By comparing the results of this new method with the so called simplified and extended analysis of Refs. [211,214], it was shown that the finite element method may result in a weaker softening behavior.

Non linear vibrations of symmetrically laminated composite shells were investigated by Amabili and Reddy [24] and Amabili $[25,217]$ based on a new higher order shear deformation theory that takes into account non linear terms involving both the normal and in plane displacements. In particular, Amabili and Reddy [24] found that the conventional higher order shear defor mation theory with von Kármán type non linearities gives inaccurate results for vibration amplitudes of about twice the shell thickness. Amabili [25] compared the non linear frequency response curves of simply supported shells obtained by (i) higher order shear deformation theory with von Kármán type non linear terms, (ii) Novozhoilov shell theory, and (iii) the new theory of Amabili Reddy. He indicated that the conventional higher order shear defor mation theory only gives accurate results for modes with high circumferential wave numbers, the Novozhilov theory gives good results for thin laminated shells and the new theory of Amabili and Reddy is preferable, if non linear vibrations of very thick shells are of interest. The comparison between these theories in predicting the non linear forced vibration responses of a thick composite shell with 0/90/90/0 lay up is shown in Fig. 7(a d). The rich non linear dynamics and internal resonances of the type 1:2 between asym metric and axisymmetric modes for a symmetric cross ply circular cylindrical shell were studied in Ref. [217]. Amabili [218] extended his previous works and studied the non linear vibrations of angle ply circular cylindrical shells by taking into account the possibility of skewed modes (modes with nodal lines not parallel to the long itudinal axis). He found an increased non linearity for a 0/30 lay up shell with respect to an identical symmetric cross ply 0/90/0 laminated shell. Moreover, Amabili [219] developed a new technique for obtaining reduced order models of angle ply circular cylindrical shells by using natural modes of vibration. In Refs. [24,25,217 219], the equations of motion were obtained by the Lagrangian approach and were solved using a pseudo arc length continuation and collo cation technique. Shen [220] performed boundary layer analysis for non linear free vibrations of anisotropic shear deformable shells and obtained hardening type non linearity for different stacking sequences. The equations of motion were obtained based on the conventional higher order shear deformation theory with von Kármán type non linearities and a singular perturbation technique was employed to solve the equations. The axisymmetric modes were neglected in the mode expansions.

Dynamic instability and buckling of composite shells were investigated by Jansen [221], Kubenko and Koval'chuk [222], Mallon [223], Mallon et al. [224], Bespalova and Urusova [225], Rahman et al. [226] and Shariyat [227,228]. Jansen [221] used Donnell's non linear shell theory and combined the Lagrangian approach with numerical time integration to study the parametric vibrations of laminated circular cylindrical shells with simply supported boundary condi tions. A two mode imperfection consisting of an axisymmetric and an asymmetric mode was taken into account and the shell was statically under axial compression, radial pressure and torsion. Dynamic buckling of isotropic and anisotropic shells under axial step loading was also simulated in Ref. [221]. Experiments on dynamic stability of composite shells were performed by Kubenko and Koval'chuk [222].The mode shapes of shells subjected to periodic excitation were studied and the effect of steady excitation on the vibration response of three layer glass/fiber reinforced plastic shells was investigated. Theory and experiments on dynamic instability of orthotropic cylindrical shells with top mass under base excitation were presented by Mallon [223] and Mallon et al. [224]. Donnell's shell theory was used and the numerical approach was based on a semi analytic technique that took into account the shell shaker interaction. The experimental results confirmed the observations of the semi analytical analysis and showed that the dynamic stability 

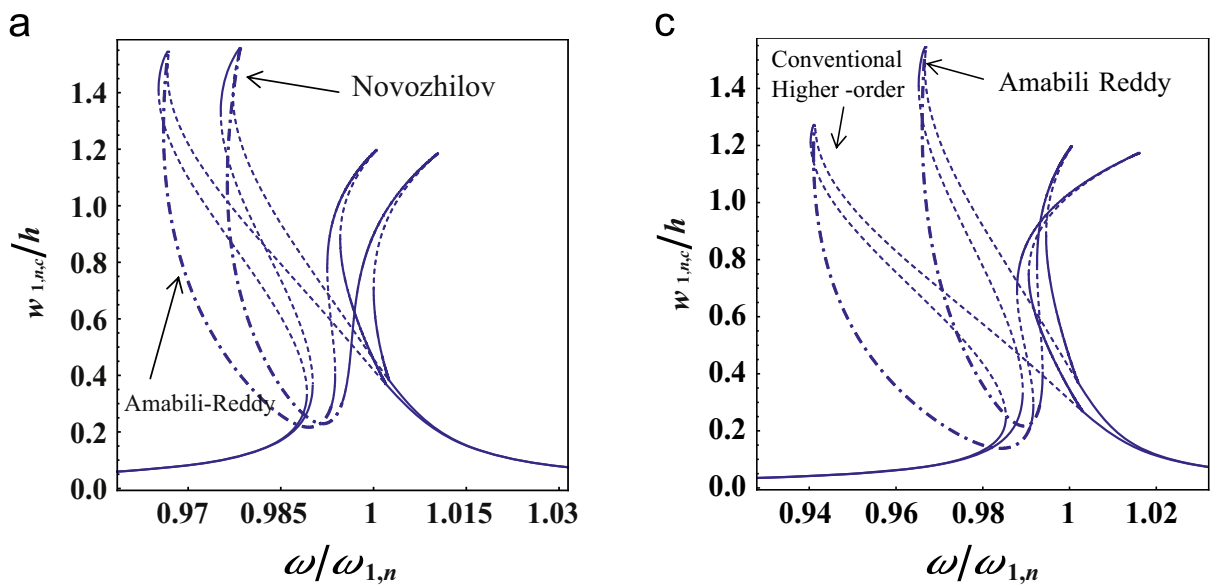

b

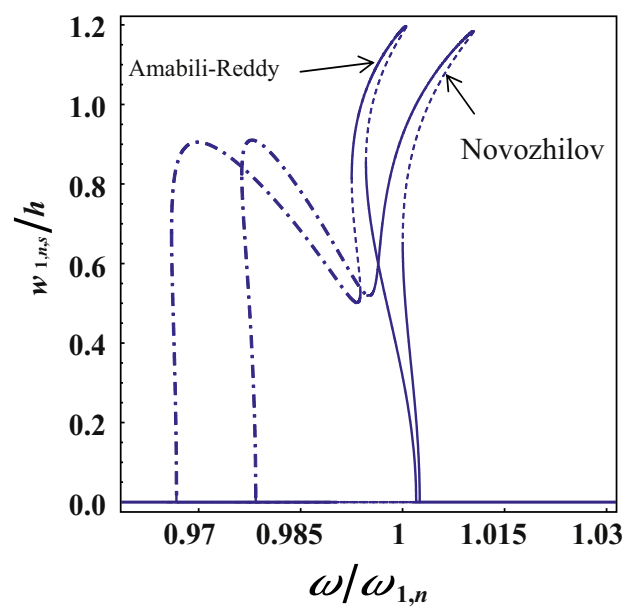

d

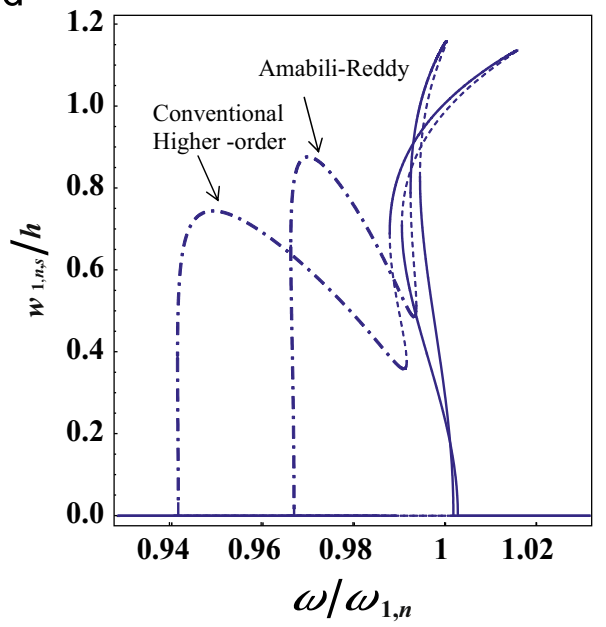

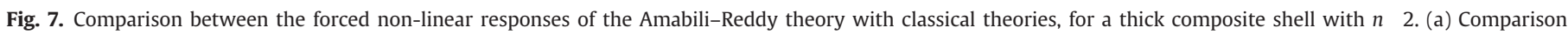

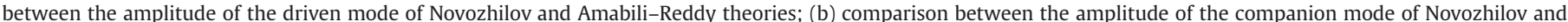

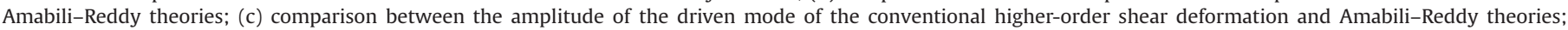

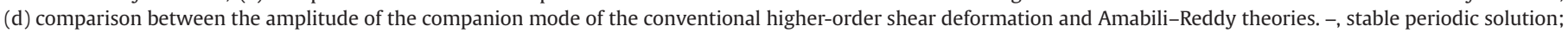
-. - , quasi-periodic stable solution; -, unstable periodic solution. From Amabili [25].

analysis of these types of structures should be focused near a resonance of relatively low frequency, corresponding to an axisym metric suspension mode, dominated by axial vibrations of the coupled system. Bespalova and Urusova [225] proposed a numerical approach to obtain the dynamic instability domains of compound shells. Rah man et al. [226] implemented a finite element perturbation method similar to [216] in order to perform a single mode dynamic buckling analysis for unstiffened and ring stiffened composite cylindrical shells. Shariyat $[227,228]$ proposed a double superposition global local for mulation inherently suitable for non linear analysis of imperfect composite and sandwich shells. In particular, in Ref. [227], viscoelastic properties of the shell were also taken into account and it was revealed that the relaxation behavior of the viscoelastic material may decrease the dynamic buckling load. In Ref. [228] it was shown that the proposed theoretical approach satisfies all the kinematic and interlaminar stress continuity conditions of composite and sandwich shells at layer interfaces. In both papers, non linear terms in transverse and in plane displacements were included in the strain displacement relations of the shell.

Non linear vibrations of sandwich shells were also investigated in Refs. [229 232]. Lugovoi et al. [229] studied the forced non stationary vibrations of sandwich circular cylindrical shells with cross ribbed cores using a finite difference scheme. Dogan and Vaicaitis [230] used Donnell's shell theory to predict the non linear response of a simply supported double wall sandwich cylindrical shell subjected to random excitation. Non linear spring dashpot models were used to characterize the behavior of the soft core of the sandwich shell. Zarutskii et al. [231] performed an extensive review on non linear vibrations of sandwich shells under moving and impulsive loads. Mohammadi and Sedaghati [232] focused on the damping characteristics of three layered sandwich cylindrical shells with thin and thick viscoelastic layers using a semi analytic finite element approach.

The effects of geometrical non linearity, rotary inertia, thermal loads and piezoelectric layers on the wave propagation of sand wich shells were discussed by Dong and Wang [233,234]. Kozlov et al. [235] studied the effect of piezoelectric layers on the active damping of forced thermo mechanical resonance vibrations of viscoelastic shells. In their paper, the effect of non linearity was due to the coupling of electromechanical and thermal fields.

\subsection{Composite panels}

Finite element formulations for non linear free vibrations of composite panels can be extensively found in the literature. Toorani [209] used a hybrid finite element approach and obtained hardening behavior for fully clamped deep orthotropic cylindrical panels. Lee et al. [236] used Donnell's theory to propose a thin laminated doubly curved panel element for the multi mode non linear free vibrations and random response of curved panels. In this paper, although 
formulations were presented for composite panels, an isotropic cylindrical panel was considered for the numerical simulations. Swamy Naidu and Sinha [237] and Nanda and Bandyopadhyay $[238,239]$ used the non linear first order shear deformation theory and an eight noded isoparametric quadrilateral element to study non linear free vibrations of doubly curved panels with simply supported boundary conditions. In particular, the effects of moisture concentration and temperature rise on the non linear response of cylindrical, spherical and hyperbolic paraboloidal panels were dis cussed in Ref. [237], the non linear to linear fundamental frequency of cylindrical panels with cut out was obtained in Ref. [238], and backbone curves of spherical panels with cut out were presented in Ref. [239]. Panda and Singh [240 242] used higher order shear deformation theory and the finite element method to obtain non linear fundamental frequency of cross ply and angle ply doubly curved panels with simply supported and clamped boundary condi tions. A nine noded isoparametric quadrilateral element was used and the transverse and in plane displacement non linear terms were retained in the strain displacement relations. In detail, non linear fundamental frequency of cylindrical and hyperbolic paraboloidal panels was presented in Ref. [240], spherical panels were studied in Ref. [241] and thermally post buckled spherical panels were exam ined in Ref. [242]. Moreover, it was shown in Refs. [241,242] that thick spherical panels exhibit hardening type behavior irrespective of the type of boundary conditions, geometry and material properties. A combination of finite element and Lindstedt Poincaré perturbation method was used by Hashemian and Jam [243] to study the vibrations of symmetrically laminated cylindrical panels. Nanda [244] and Nanda and Pradyumna [245] used a combination of finite element and direct iteration method to obtain the non linear fundamental frequency of laminated cylindrical and spherical panels. Piezoelectric and thermal effects were discussed in Ref. [244], whereas hygrothermal and geometric imperfection effects were investigated in Ref. [245]. In both papers, the finite element formula tions were based on higher order shear deformation theory with von Kármán type non linear terms and panels had simply supported and clamped boundary conditions. Panda and Singh [246] used the same theory and finite element formulation of Ref. [242] to obtain non linear fundamental frequency of thermally post buckled doubly curved panels. Mohammdai and Sedaghati [247] discussed the effects of different boundary conditions, small and large displacements, core thickness ratio and electric field intensity on the non linear vibration and damping characteristics of sandwich cylindrical panels with a constrained electrorheological fluid. Finite element formulations similar to Refs. [242,246] were used by Panda and Singh [248] to obtain the non linear fundamental frequency of laminated spherical panels with embedded shape memory alloy fibers.

Non linear free vibrations of composite panels were also investigated with solution techniques other than the finite element method. Kurpa [249] used the first order shear deforma tion theory and the R functions method to obtain the backbone curves of simply supported multi layer cylindrical panels with complex bases. The effect of variable thickness was introduced in Ref. [250]. Yazdi [251] used Donnell's non linear shallow shell theory and Galerkin single mode approximation together with the homotopy perturbation method to obtain the backbone curves of cross ply doubly curved panels.

Non linear forced vibrations of composite panels subjected to transverse harmonic loads were investigated in Refs. [26,252 256]. Experiments on non linear vibrations of graphite/epoxy cylindrical panels were carried out by Amabili et al. [252]. A plain weave fabrics and a three layer 90/0/ 90 panel were tested and the trend of non linearity was characterized following a stepped sine tech nique and by increasing and decreasing the excitation frequency in very small steps in the frequency neighborhood of the lowest natural frequencies. The results showed strong softening behavior.
Adam [253,254] studied non linear flexural vibrations of multi layered doubly curved panels based on a first order layer wise theory. In Ref. [253] the effect of initial imperfections was discussed and it was shown that, depending on the amplitude of the imperfection, a thick laminated panel may exhibit hardening or softening type non linearity, or even a combination of both. The effects of initial curvature and transverse shear flexibility on the non linear frequency amplitude response of panels composed of thick layers were examined in Ref. [254]. Abe et al. [255] used the first order shear deformation theory to study the one to one internal resonance between the second and third asymmetric vibration modes of fully clamped anti symmetric angle ply doubly curved panels. A three degree of freedom model was obtained by applying the Galerkin method and was solved by a shooting technique. Ribeiro [256] discussed the influence of in plane mem brane inertia and shear deformation on non linear forced vibrations of clamped cylindrical panels. Non linear first order shear deforma tion theory was used and the $p$ version finite element method with hierarchic basis functions was employed to obtain the non linear governing equations that were then integrated by using Newmark's technique. Alijani and Amabili [26] used the new theory of Amabili and Reddy [24] to study the rich non linear dynamics and diverse internal resonances of thick and deep laminated circular cylindrical panels. In particular, 1:1, 2:1, 3:1 and 1:1:2 internal resonances were found between different modes of laminated panels and quasi periodic, intermittent and chaotic responses were observed in the vicinity of the internal resonances. Moreover, it was illustrated that laminates with stronger couplings between in plane and shear strains exhibit stronger softening and/or hardening behavior.

Non linear vibrations of curved panels subjected to transverse non periodic dynamic loads were investigated by Shul'ga and Meish [257], Lentzen et al. [258], Ribeiro and Jansen [259], Nanda and Bandyopadhyay [260], Li et al. [261], Nanda [244] and Nanda and Pradyumna [245]. Shul'ga and Meish [257] used the first order shear deformation theory and a finite difference scheme to obtain the dynamic response of three layer spherical and ellipsoidal panels to impulsive loads. Lentzen et al. [258] discussed the non linear dynamic response of clamped semicircular panels with piezoelectric layers subjected to line loads using the finite element method. Ribeiro and Jansen [259] used the non linear first order shear deformation theory and the $p$ version finite element method to study the influence of temperature and fiber orientation on the dynamics of fully clamped panels subjected to uniformly distributed impulse. They found that fiber orientations have a strong effect on the thermo mechanical behavior of panels and the response of thick panels is less influenced by the changes of temperature compared to thin ones. Fig. 8(a c) shows the effect of fiber orientation on a symmetrically laminated circular cylindrical panel subjected to a uniformly distributed rectangular impulse. Nanda and Bandyopad hyay [260] investigated the non linear transient response of cylind rical and spherical panels with cut out using the finite element method. Non linear first order shear deformation theory was used and the responses of the panel to step and half sine loads were obtained. The non linear response of shallow sandwich panels subjected to blast loading was obtained by Li et al. [261] using a higher order shear deformation theory suitable for panels with compressible core. Nanda [244] and Nanda and Pradyumna [245] used the Newmark average acceleration method with the modified Newton Raphson iteration scheme to perform a transient analysis on piezo laminated and imperfect composite panels, respectively.

Dynamic buckling of composite panels was investigated by $\mathrm{Fu}$ et al. [262] and Shariyat [227,228]. Fu et al. [262] used the non linear first order shear deformation theory to study the dynamic buckling of damaged thick spherical panels under impact load. Shariyat [227,228] provided various examples for the dynamic buckling of perfect, imperfect, thick, relatively thin, multilayered 
a

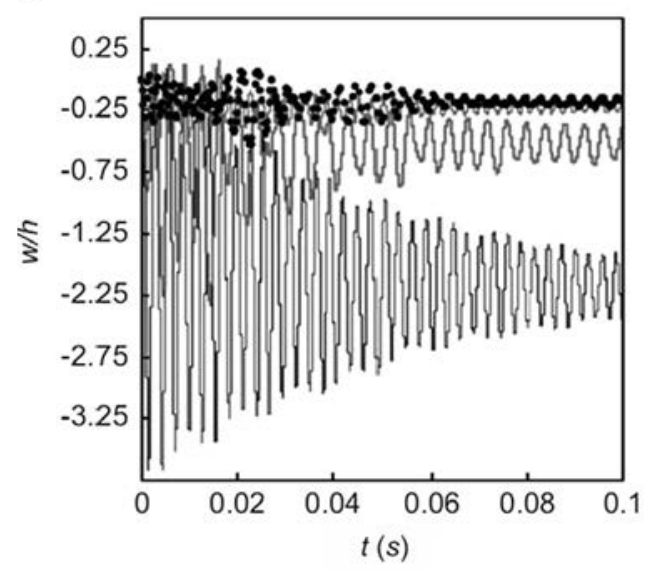

b

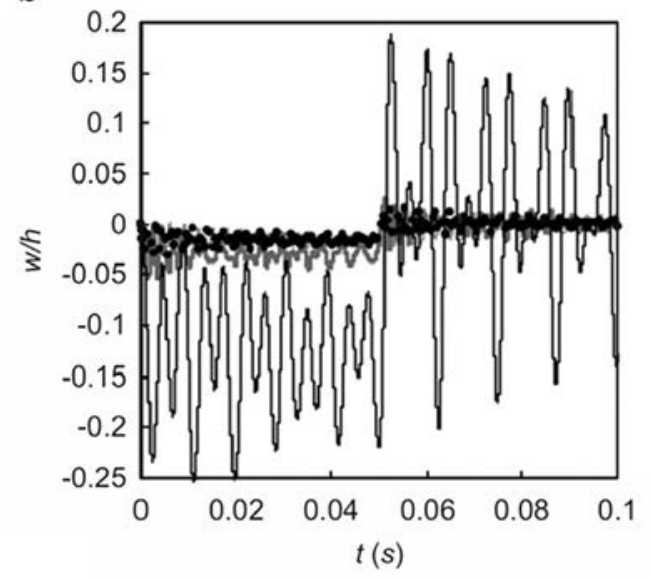

C

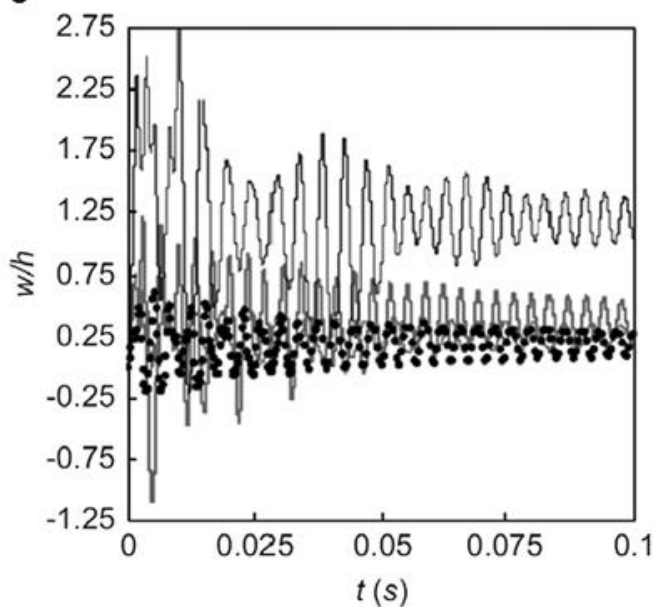

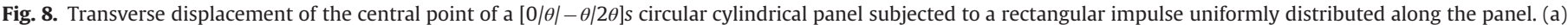

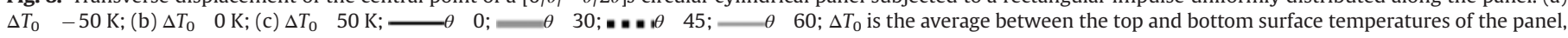
and $\theta$ is the orientation angle. From Ribeiro and Jansen [259].

or sandwich panels with stiff and flexible cores under suddenly applied thermal and mechanical loads.

Dynamic instability of laminated cylindrical and spherical panels with piezoelectric layers subjected to in plane periodic load and electric load was examined by Pradyumna and Gupta [263] using the finite element method. The theoretical formula tion was based on the non linear first order shear deformation theory. It was found that the effect of the location of piezoelectric layers is significant at higher vibration amplitudes and marginal at lower amplitudes. Active damping of geometrically non linear vibrations of laminated curved panels with piezoelectric layers was studied by Sarangi and Ray [264,265], Karnaukhov et al. [266] and Shivakumar et al. [267].

\subsection{Fluid structure interaction in composite shells}

Fluid structure interaction of composite shells and panels is rarely investigated in the literature. The effects of material ortho tropy on the non linear vibrations and dynamic instability of circular cylindrical shells in contact with flowing fluid were investigated by del Prado et al. [268]. Donnell's non linear shal low shell theory was used and the fluid was assumed to be non viscous and incompressible. The equations of motion were obtained by the Galerkin approach and were solved by a Runge Kutta technique. An eight degree of freedom model was consid ered that included the driven, companion, gyroscopic (i.e. the two modes with two longitudinal half waves) and four axisymmetric modes. Lakiza [269] studied the non linear vibrations of fluid filled glass fiber reinforced cylindrical shells subjected to radial two frequency excitation. Non linear stability of composite shells in subsonic air flow under radial harmonic excitation was inves tigated by Li and Yao [270]. Their analysis was based on a rough single mode Galerkin approximation and the method of multiple scales.

Geometrically non linear finite element formulation for aero thermoelastic analysis of piezo laminated cylindrical panels was given by Oh and Lee [271]. A multi field layer wise theory was used to study the supersonic flutter characteristics of the compo site panel. It was found that the critical aerodynamic pressure of piezo laminated cylindrical panels increases with the increase of surface temperature. Shin et al. [272] used the finite element method to study the non linear flutter of aero thermally buckled laminated cylindrical panels. An arc length method together with a non linear iteration scheme was used to solve the equations of motion. The flutter boundaries of various damped composite panels with different damping treatments, including constrained and sandwiched viscoelastic layers, were investigated by varying the thermal loading.

Besides common structural applications, composite shell the ories are often used to model arteries conveying flow in biome chanics. As a first attempt to study the non linear response of human aortic segments, Amabili et al. [273] used the laminated circular cylindrical shell model and the non linear Sanders Koiter shell theory to study the non linear stability of an aortic segment 
under steady flow conditions. The aorta was modeled as a three layer composite shell, representing the tunica intima, media and adventitia, and the fluid model contained the unsteady effects of linear potential flow theory and the steady viscous effects obtained from the time averaged Navier Stokes equations. It was shown that the aortic segment loses stability by divergence with deformation of the cross section at a critical flow velocity for a given static pressure, exhibiting a strong subcritical behavior with partial or total collapse of the inner wall.

\section{Non-linear vibrations of functionally graded shells}

In this section, papers that have dealt with geometrically non linear vibrations of functionally graded (FG) shells and panels are reviewed. FGMs are inhomogeneous composites fabricated from a mixture of metal and ceramic with smooth and continuous variation of material properties through the thickness. Compared to isotropic and laminated shells they have reduced thermal stresses and stress concentrations and have the capability of withstanding high temperature gradient environments without losing structural integrity. These advantages, together with their high strength and light weight, have made functionally graded materials a suitable replacement for conventional materials in many industrial applications as thermal barriers. Therefore, study ing the vibration characteristics of FG structures under large amplitude thermo mechanical loads has become of paramount importance in the last decade.

\subsection{Closed FGM shells}

Darabi et al. [274] studied the dynamic stability of FG shells at constant temperature subjected to periodic axial loads. Donnell's non linear shallow shell theory was used and a combination of the Galerkin and the Bolotin methods was employed to solve the equations of motion. Axisymmetric modes were neglected in the displacement expansions, and therefore only hardening type behavior was obtained.

Mahmoudkhani et al. [275] used multi mode Galerkin approx imation and the method of multiple scales to study the primary resonance response of simply supported cylindrical shells. Don nell's non linear shallow shell theory was used and the displace ment expansion included driven, companion and axisymmetric modes. The effect of FG volume fraction exponent, length to radius and thickness to radius ratios on the trend of non linearity was discussed; however, temperature effects were neglected.

Non linear free vibrations of FG shells surrounded by Pasternak foundation were investigated by Shen [276]. Higher order shear deformation theory was used, but in a version that makes use of Airy's stress function to condensate in plane displacements, so it retains transverse and rotary inertia but neglects in plane inertia. The effective material properties of the FG shell were obtained using the rule of mixtures (Voigt model) and the Mori Tanaka micromechanics scheme, which is usually applicable to regions of the graded microstructure with well defined continuous matrix and discontin uous particulate phase. It was assumed that temperature varies only in the thickness direction and the temperature distribution was obtained by solving a one dimensional steady state heat transfer equation. A two step perturbation approach was utilized to obtain the non linear frequencies of the shell, neglecting the effect of axisymmetric modes. Hardening type non linearity was predicted for thick shells and it was found that, in most cases, Voigt and Mori Tanaka models have the same levels of accuracy in predicting the non linear response of FGM shells. Shen and Xiang [277] also used the same theory and methodology as described in [276] to study non linear vibrations of nano tube FG reinforced shells.
Bich and Xuan Nguyen [278] studied non linear free and forced vibrations of FG shells under pre load compression at constant temperature. Based on Donnell's shell theory and neglecting in plane inertia, they employed a single mode Galerkin approxima tion to discretize the transverse displacement. An averaging method was used to obtain the backbone curves whereas a Runge Kutta scheme was utilized to obtain the frequency response curves. The effects of axisymmetric (i.e. axisymmetric shell contraction during vibrations) and companion (i.e. 1:1 inter nal resonance) modes were neglected, and it is well known that this gives wrong results. Results are presented for a mode with 3 circumferential waves, when it has been established that in plane inertia should be retained at least for modes up to 3 circum ferential waves (the condition $1 / n^{2} \ll 1$ must be satisfied in order to neglect in plane inertia, where $n$ is the number of circumfer ential waves).

Non linear dynamics and bifurcations of FG shells subjected to large amplitude harmonic radial loads were investigated by Hao et al. [279,280] and Zhang et al. [281]. First order shear deforma tion theory was used and the Galerkin approach was employed to obtain a two degree of freedom non linear system that was solved by a fourth order Runge Kutta method in order to obtain bifurca tion diagrams, Poincaré sections and time responses of the shell. In all three papers, the effect of axisymmetric and companion modes was neglected, again with erroneous results.

Sheng and Wang [282] used the first order shear deformation theory and the Galerkin multi mode approach together with the method of multiple scales to study the effects of temperature change, thickness ratio and volume fraction exponent on the non linear vibration response of the shell. Companion modes were neglected but the transverse displacement of the shell was expanded in the form of Ref. [64] and included axisymmetric and driven modes.

Rafiee et al. [283,284] studied non linear vibrations of FG shells with piezoelectric layers in thermal environments and subjected to supersonic airflow. The method of multiple scales was used and the analysis was based on a single degree of freedom approxima tion that showed hardening type behavior. Single mode Galerkin approximation was also performed by Najafov and Sofiyev [285] to examine the non linear vibrations of FG truncated conical shells at constant temperature. Dynamic buckling of FG conical shells subjected to time dependent compressive axial loads was inves tigated by Deniz and Sofiyev [286] using the Galerkin and the Runge Kutta methods.

Recently, Strozzi and Pellicano [287] used the Sanders Koiter non linear shell theory and a Lagrangian approach to investigate the forced non linear vibrations of FG circular cylindrical shells subjected to radial harmonic excitation, neglecting the thermal effects. Simply supported, clamped and free boundary conditions were considered and the shell displacements were expanded by using Chebyshev polynomials. By considering different multi dimensional models with sufficient asymmetric and axisymmetric modes, a convergence study was carried out and it was found that very short and thick, and sufficiently long and thin FG shells exhibit hardening type non linearity.

\subsection{FGM panels}

Liew et al. [288] investigated the non linear free vibrations of a three layer coating FGM substrate cylindrical panel with different boundary conditions and subjected to a temperature gradient across the thickness due to steady heat conduction. First order shear deformation theory was used and the numerical solution was obtained by using the differential quadrature method. The effects of geometry, volume fraction exponent, temperature and 
boundary conditions on the non linear fundamental frequency of cylindrical panels were discussed.

Pradyumna et al. [289] used higher order finite element formulation to examine the non linear transient vibrations of FG doubly curved panels with rectangular base. An eight noded $\mathrm{C}^{0}$ element with nine degrees of freedom at each node was used and the transient response of the system was calculated by the New mark method. Pradyumna and Nanda [290] also extended their work by including initial geometric imperfections and thermal effects.

Chorfi and Houmat [291] investigated the non linear funda mental frequency of doubly curved shallow shells with clamped boundary conditions using the $p$ version finite element method along with the harmonic balance method. They showed that FG thick panels present a hardening behavior and discussed the effect of volume fraction exponent on that behavior. In their analysis, temperature effects on system response were neglected.

A comprehensive study on the non linear vibrations of thin simply supported FG doubly curved panels at constant tempera ture was undertaken by Alijani et al. [292]. By deriving the equations of motion in terms of transverse deflection and Airy stress function as the only dependent variables, and in order to overcome the shortcomings of low order Galerkin discretization, the equations of motion were discretized by the full basis Galerkin approach using the complete set of eigen functions of the associated linear system to obtain a system of infinite non linear ordinary differential equations with quadratic and cubic non linearities. Then, using the method of multiple scales, the effects of curvature and FGM volume fraction exponent index on the non linear behavior were investigated when (i) the excitation fre quency is close to the fundamental frequency (primary resonance case) and (ii) twice that frequency (sub harmonic resonance case). Subsequently, using a numerical scheme, internal resonance and chaotic vibrations were studied. Later, Alijani et al. [293] extended their work by including the thermal effects and discussed the non linear vibration characteristics of doubly curved panels with conventional non linear higher order shear deformation and Amabili Reddy non linear higher order shear deformation the ories [24]. The analysis was based on multi mode Lagrangian approach and the pseudo arc length continuation scheme was used to perform bifurcation analysis. It was shown that, for thin FG spherical panels, the response is softening, turning to hardening behavior for large vibration amplitudes. However, thick spherical panels exhibit hardening non linearity. Moreover, it was revealed that the temperature plays different roles depending on the thickness and shallowness of the panel. Alijani and Amabili [294] also studied the chaotic vibrations and internal resonances of doubly curved FG panels. To classify the complex dynamics, the maximum Lyapunov exponent and the full spectrum of Lyapunov exponents were calculated for several multi dimensional models.

Bich et al. [295,296] and Duc [297] investigated the non linear vibrations and dynamic buckling of eccentrically stiffened FG panels, neglecting the temperature effects. In the three papers, the equations of motion were obtained using Donnell's shell theory. In plane inertia was neglected and the problem was reduced to a single degree of freedom non linear equation that was solved using a Runge Kutta technique. In particular, in Ref. [295] only cylindrical panels were studied, in Ref. [296] the work was extended to doubly curved panels and in Ref. [297] the panel was assumed to be on elastic foundation.

\section{Non-linear vibrations of hyperelastic shells}

Studies dealing with the non linear dynamic behavior of shells made of rubber like materials were primarily discussed in the 1960s and continue to the present. In fact, rubber like materials are capable of withstanding large deformations, and therefore, in addition to geometrical non linearities (non linear strain displa cement relations), physical or material non linearities based on hyperelastic models (non linear stress strain relations) should also be retained in their numerical models [298]. In particular, strain invariant constitutive models such as neo Hookean, Mooney Rivlin, Ogden and Fung models should be used to capture the non linear behavior of hyperelastic materials.

Owing to the complicated nature of these models, reported studies on the dynamic problems of hyperelastic shells are very scarce. Early investigations on the large amplitude radial oscillations of circular cylindrical shells made of incompressible hyperelastic materials were performed by Knowles [299,300]. In these works the governing equation of motion was an autonomous single degree of freedom equation and the free vibrations caused by suddenly applied and subsequently maintained uniform pressures were studied. Later, Shahinpoor and Nowinski [301] and Shahinpoor and Balakrishnan [302] studied the large amplitude vibrations of circular cylindrical shells made of incompressible materials and Mooney Rivlin type rubber. In particular, an analytic solution was presented in Ref. [301] and a numerical study based on a fourth order Runge Kutta techni que was employed in Ref. [302] for the single degree of freedom model previously presented by Knowles [299]. Free vibrations of neo Hookean circular cylindrical shells were studied by Wang and Ertepinar [303] and Ertepinar and Akay [304]. The finite amplitude vibrations of non linearly elastic incompressible spherical and cylindrical shells were examined mathematically by Calderer [305]. Akyuz and Ertepinar [306] investigated the stability and asymmetric vibrations of pressurized compressible hyperelastic cylindrical shells. The hyperelastic model that they used was based on Levinson Burgess polynomial compressible material, which is coincident with the neo Hookean law for incompressible materials. In order to solve the problem, they first found the static equilibrium state of the solution for finite deformations, and then used the small perturba tions theory to investigate the free vibrations of the shell around the equilibrium state. A shooting method was also utilized to solve the governing equations of motion and the numerical solutions were found to be in good agreement with the finite element results.

Since 2003, new researches performed on hyperelastic shells have mostly been focused on the stability and buckling of elastic shells subjected to external/internal pressure. For instance, based on the non linear theory of elasticity, Zhu et al. [307] discussed the asymmetric bifurcations of thick shells made of isotropic neo Hookean materials and under combined axial loading and external pressure. Zhu et al. [308] extended their previous work [307] by studying the non linear axisymmetric deformations of incompressi ble hyperelastic shells subjected to zero displacements on their ends and pressure on their external lateral surfaces. Recently, the same authors [309] investigated the three dimensional non linear buck ling of pressurized circular cylindrical shells using a finite element algorithm and highlighted the post buckling deformations.

Concerning non linear vibrations of hyperelastic shells, the only works available since 2003 are those of Sansour et al. [176], Yuan et al. [310], Yong et al. [151], He et al. [152] and Ren [311,312]. In particular, Sansour et al. [176] constructed an energy momentum method to study the non linear dynamics of shells in the framework of the finite element method and discussed the large amplitude response of shells considering non linear constitutive laws. Yuan et al. [310] studied the dynamic inflation of infinitely long cylindrical tubes subjected to periodic step pressures on the inner surface. The incompressible Ogden material model was used and the strain energy density function was reduced to neo Hookean type. Sensitiv ity of the dynamics to the material parameters, geometry and the applied pressures was discussed. In Refs. $[151,152]$ the dynamics of dielectric incompressible neo Hookean spherical and cylindrical 
shells was studied, respectively, and it was found that the system is more stable when the thickness of the shell increases. Ren [311] determined the critical applied pressure of an incompressible neo Hookean circular cylindrical shell subjected to periodic or suddenly applied constant load on the inner surface. He found that when the pressure is lower than the critical value, the fixed point is a center surrounded by a homoclinic orbit, and the shell undergoes non linear periodic oscillations. On the other hand, when the pressure is larger than the critical value, the fixed point becomes an unstable saddle node, and the shell's displacements will ultimately diverge. The same author [312] also discussed the dynamics of neo Hookean spherical shells, and by plotting Poincaré maps and phase plane plots showed that the shell may undergo quasi periodic oscillations once it is subjected to periodic internal pressure.

\section{Experiments on large-amplitude vibrations of shells}

Although a vast literature is available on geometrically non linear vibrations of shells and panels, the experimental investiga tions are quite scarce. A complete list of experimental works on non linear vibrations and fluid structure interactions of shell structures before 2003 can be found in the review study of Amabili and Païdoussis [5].

Comprehensive experimental analysis on large amplitude vibra tions of empty and fluid filled circular cylindrical shells subjected to radial harmonic excitation was carried out by Amabili [56]. Pellicano and Avramov [94] and Pellicano [95] performed experi ments on cylindrical shells connected to a rigid disk and under base excitation to obtain damping ratios for numerical simulations. Pellicano [96] extended his previous experimental tests by follow ing a stepped sine approach and obtained the experimental stabi lity diagrams of a shell connected to top mass. Experiments on the stability of shells subjected to fluid flow were carried out by Karagiozis et al. [121,122,124,125]. Shells having clamped ends and subjected to external airflow and internal water flow were tested. Lakiza [313,314] performed experiments on non linear vibrations of compound (truncated cone with spherical shell) and circular cylindrical shells with a gas liquid medium, respectively.

Experiments on non linear vibrations of cylindrical panels have been conducted by Amabili et al. [157] and Amabili [160]. Geome trically non linear dynamic behavior of cylindrical panels with complex base subjected to transverse excitation has been investi gated by Kurpa et al. [170]. Experimental investigation of chaos in cylindrical and doubly curved panels has been carried out by Nagai et al. [180,184] and Maruyama et al. [183]. Thomas et al. [192] performed experimental tests on a free edge spherical cap in order to validate the occurrence of a rare modal interaction coupling.

Kubenko and Koval'chuk [222] studied the dynamic stability of a three layer composite shell experimentally. Mallon [223] and Mallon et al. [224] performed experiments on an orthotropic circular cylindrical shell subjected to base (seismic like) excitation. The only available experimental work on geometrically non linear vibrations of composite cylindrical panels has been conducted by Amabili et al. [252].

\section{Conclusions}

The purpose of this review is (i) to help researchers working in the field of non linear vibrations of shell structures with the required references for their research and (ii) to give ideas of what researches can be undertaken and what researches could be of paramount importance. In writing this review the authors have tried to be as complete as possible. However, it is plausible that some studies are missed out. The authors apologize to the authors of papers that have accidentally been left out of this review.

It must be observed that the research performed on large amplitude vibrations of shell structures, specifically for those made of advanced materials, is still far from being considered well established. Regarding isotropic materials, the research on geome trically non linear vibrations of conical shells and panels is still in the primary stage and is hoped to be enhanced in the near future. In case of composite shells and panels (i) experimental investiga tions and (ii) non linear fluid structure interactions can be rarely found in the literature, and therefore need particular attention. For FG shells, most of the studies that are published neglect the fundamental and preliminary features of shell structures, such as the effect of axisymmetric modes in closed shells and in plane modes in curved panels. Thus, in order to clarify the non linear dynamic behavior of this type of shells, a more careful investigation is required. Finally, in case of hyperelastic shells, no comprehensive research has yet been conducted on the dynamic instability and non linear vibrations of highly pressurized shells taking into account shear deformation and thickness variation effects.

\section{Acknowledgments}

The authors acknowledge the financial support of the PSR SIIRI program of Québec, NSERC Discovery Grant, Canada Research Chair, Canada Foundation for Innovation (LOF) programs of Canada and European Commission FP7/PEOPLE/MARIECURIE SUPERPA NELS (Strengthening and Upholding the Performances of new Engineered Research PANELS).

\section{References}

[1] A.W. Leissa, Vibration of Shells, NASA-SP-288, LC-77-186367, 1973.

[2] D.A. Evensen, Nonlinear vibrations of circular cylindrical shells, in: Y.C. Fung, E.E. Sechler (Eds.), Thin-Shell Structures: Theory, Experiment and Design, Prentice-Hall, New York, 1974.

[3] V.D. Kubenko, P.S. Koval'chuk, Nonlinear problems of the vibration of thin shells (review), International Applied Mechanics 34 (1998) 703-728.

[4] V.D. Kubenko, P.S. Koval'chuk, Nonlinear problems of the dynamics of elastic shells partially filled with a liquid, International Applied Mechanics 36 (2000) 421-448

[5] M. Amabili, M.P. Païdoussis, Review of studies on geometrically nonlinear vibrations and dynamics of circular cylindrical shells and panels, with and without fluid-structure interaction, Applied Mechanics Reviews 56 (2003) 349-381.

[6] F. Moussaoui, R. Benamar, Non-linear vibrations of shell-type structures: a review with bibliography, Journal of Sound and Vibration 255 (2002) 161-184.

[7] M. Amabili, Nonlinear Vibrations and Stability of Shells and Plates, Cambridge University Press, New York, 2008.

[8] E.H. Donnell, A new theory for the buckling of thin cylinders under axial compression and bending, Transactions of the ASME 56 (1934) 795-806.

[9] V.V. Novozhilov, Foundations of the Nonlinear Theory of Elasticity, Graylock press, New York, 1953.

[10] L.J. Sanders, Nonlinear theories for thin shells, Quarterly of Applied Mathematics 21 (1963) 21-36.

[11] W.T. Koiter, On the nonlinear theory of thin elastic shells, Proceedings Koniklijke Nederlands Akademie van Wetenschappen B69 (1966) 1-54.

[12] J.H. Ginsberg, Large-amplitude forced vibrations of simply supported thin cylindrical shells, ASME Journal of Applied Mechanics 40 (1973) 471-477.

[13] J.N. Reddy, Mechanics of Laminated Composite Plates and Shells: Theory and Analysis, CRC Press, Boca Raton, 2004

[14] E. Carrera, S. Brischetto, P. Nali, Plates and Shells for Smart Structures: Classical and Advanced Theories for Modeling and Analysis, John Wiley \& Sons, UK, 2011.

[15] E. Carrera, Theories and finite elements for multilayered, anisotropic composite plates and shells, Archives of Computational Methods in Engineering 9 (2002) 87-140.

[16] E. Carrera, Historical review of zig-zag theories for multilayered plates and shells, Applied Mechanics Reviews 56 (2003) 287-308.

[17] J.N. Reddy, R. Arciniega, Shear deformation plate and shell theories: from Stavsky to present, Mechanics of Advanced Materials and Structures 11 (2004) 535-582.

[18] J.N. Reddy, K. Chandrashekhara, Geometrically non-linear transient analysis of laminated, doubly curved shells, International Journal of Non-Linear Mechanics 20 (1985) 79-90. 
[19] L. Librescu, Refined geometrically non-linear theories of anisotropic laminated shells, Quarterly of Applied Mathematics 45 (1987) 1-22.

[20] S.T. Dennis, A.N. Palazotto, Large displacement and rotational formulation for laminated shells including parabolic transverse shear, International Journal of Non-Linear Mechanics 25 (1990) 67-85.

[21] A.N. Palazotto, S.T. Dennis, Nonlinear Analysis of Shell Structures, AIAA Educational Series, Washington DC, 1992.

[22] J. Hohe, L. Librescu, A nonlinear theory for doubly curved anisotropic sandwich shells with transversely compressible core, International Journal of Solids and Structures 40 (2003) 1059-1088.

[23] R.A. Chaudhuri, A nonlinear zigzag theory for finite element analysis of highly shear-deformable laminated anisotropic shells, Composite Structures 85 (2008) 350-359.

[24] M. Amabili, J.N. Reddy, A new non-linear higher-order shear deformation theory for large-amplitude vibrations of laminated doubly curved shells, International Journal of Non-Linear Mechanics 45 (2010) 409-418.

[25] M. Amabili, Nonlinear vibrations of laminated circular cylindrical shells: comparison of different shell theories, Composite Structures 94 (2011) 207-220.

[26] F. Alijani, M. Amabili, Nonlinear vibrations of thick laminated circular cylindrical panels, Composite Structures 96 (2013) 643-660.

[27] M. Amabili, A new non-linear higher order shear deformation theory with thickness variation for large-amplitude vibrations of laminated doubly curved shells, Journal of Sound and Vibration 332 (2013) 4620-4640.

[28] M. Amabili, A non-linear higher-order thickness variation and shear deformation theory for large-amplitude vibrations of laminated doubly curved shells, International Journal of Non-Linear Mechanics 58 (2014) 57-75.

[29] H. Parisch, A continuum-based shell theory for non-linear applications, International Journal for Numerical Methods in Engineering 38 (1995) 1855-1883.

[30] C. Sansour, A theory and finite element formulation of shells at finite deformations involving thickness change: circumventing the use of a rotation tensor, Archive of Applied Mechanics 65 (1995) 194-216.

[31] E. Carrera, S. Brischetto, M. Cinefra, M. Soave, Effects of thickness stretching in functionally graded plates and shells, Composites Part B: Engineering 42 (2011) 123-133.

[32] A.J.M. Ferreira, E. Carrera, M. Cinefra, C.M.C. Roque, Analysis of laminated doubly-curved shells by a layerwise theory and radial basis functions collocation, accounting for through-the-thickness deformations, Computational Mechanics 48 (2011) 13-25.

[33] V. Eremeyev, W. Pietraszkiewicz, The nonlinear theory of elastic shells with phase transitions, Journal of Elasticity 74 (2004) 67-86.

[34] S. Opoka, W. Pietraszkiewicz, Intrinsic equations for non-linear deformation and stability of thin elastic shells, International Journal of Solids and Structures 41 (2004) 3275-3292.

[35] R.A. Arciniega, J.N. Reddy, Tensor-based finite element formulation for geometrically nonlinear analysis of shell structures, Computer Methods in Applied Mechanics and Engineering 196 (2007) 1048-1073.

[36] S. Opoka, W. Pietraszkiewicz, On modified displacement version of the nonlinear theory of thin shells, International Journal of Solids and Structures 46 (2009) 3103-3110.

[37] V.L. Berdichevsky, Nonlinear theory of hard-skin plates and shells, International Journal of Engineering Science 48 (2010) 357-369.

[38] S. Xiaoqin, L. Kaitai, M. Yang, The modified model of Koiter's type for nonlinearly elastic shell, Applied Mathematical Modelling 34 (2010) 3527-3535.

[39] W. Pietraszkiewicz, On exact expressions of the bending tensor in the nonlinear theory of thin shells, Applied Mathematical Modelling 36 (2012) 1821-1824.

[40] D. Steigmann, Koiter's shell theory from the perspective of threedimensional nonlinear elasticity, Journal of Elasticity 111 (2013) 91-107.

[41] V. Eremeyev, Nonlinear micropolar shells: theory and applications, in: W. Pietraszkiewicz, C. Szymczak (Eds.), Shell Structures: Theory and Applications, Taylor \& Francis, London, 2005, pp. 11-18.

[42] V. Eremeyev, W. Pietraszkiewicz, Local symmetry group in the general theory of elastic shells, Journal of Elasticity 85 (2006) 125-152.

[43] V. Eremeyev, L. Zubov, On constitutive inequalities in nonlinear theory of elastic shells, ZAMM - Journal of Applied Mathematics and Mechanics Zeitschrift für Angewandte Mathematik und Mechanik 87 (2007) 94-101.[44] J.

Altenbach, H. Altenbach, V. Eremeyev, On generalized Cosserat-type theories of plates and shells: a short review and bibliography, Archive of Applied Mechanics 80 (2010) 73-92.

[45] H. Altenbach, V.A. Eremeyev, On the shell theory on the nanoscale with surface stresses, International Journal of Engineering Science 49 (2011) 1294-1301.

[46] K.A. Lazopoulos, A.K. Lazopoulos, Nonlinear strain gradient elastic thin shallow shells, European Journal of Mechanics - A/Solids 30 (2011) 286-292.

[47] D.A. Evensen, Nonlinear Flexural Vibrations of Thin-walled Circular Cylinders, NASA TN D-4090, 1967.

[48] D.A. Evensen, Nonlinear vibrations of an infinitely long cylindrical shell, AIAA Journal 6 (1968) 1401-1403.

[49] E.H. Dowell, C.S. Ventres, Modal equations for the nonlinear flexural vibrations of a cylindrical shell, International Journal of Solids and Structures 4 (1968) 975-991.

[50] S. Atluri, A perturbation analysis of non-linear free flexural vibrations of a circular cylindrical shell, International Journal of Solids and Structures 8 (1972) 549-569.
[51] D.A. Evensen, Some observations on the nonlinear vibrations of thin cylindrical shells, AIAA Journal 1 (1963) 2857-2858.

[52] M.D. Olson, Some experimental observations on the nonlinear vibrations of cylindrical shells, AIAA Journal 3 (1965) 1775-1777.

[53] T.K. Varadan, G. Prathap, H.V. Ramani, Nonlinear free flexural vibration of thin circular cylindrical shells, AIAA Journal 27 (1989) 1303-1304

[54] M. Amabili, F. Pellicano, M.P. Païdoussis, Non-linear dynamics and stability of circular cylindrical shells containing flowing fluid, part II: large-amplitude vibrations without flow, Journal of Sound and Vibration 228 (1999) 1103-1124.

[55] M. Amabili, F. Pellicano, M.P. Païdoussis, Non-linear dynamics and stability of circular cylindrical shells containing flowing fluid. Part III: truncation effect without flow and experiments, Journal of Sound and Vibration 237 (2000) 617-640.

[56] M. Amabili, Theory and experiments for large-amplitude vibrations of empty and fluid-filled circular cylindrical shells with imperfections, Journal of Sound and Vibration 262 (2003) 921-975.

[57] M. Amabili, A comparison of shell theories for large-amplitude vibrations of circular cylindrical shells: Lagrangian approach, Journal of Sound and Vibration 264 (2003) 1091-1125.

[58] A.A. Popov, Auto-parametric resonance in thin cylindrical shells using the slow fluctuation method, Thin-Walled Structures 42 (2004) 475-495.

[59] A.A. Popov, The application of Hamiltonian dynamics and averaging to nonlinear shell vibration, Computers \& Structures 82 (2004) 2659-2670.

[60] K.V. Avramov, Nonlinear forced vibrations of a cylindrical shell with two internal resonances, International Applied Mechanics 42 (2006) 169-175.[61]

K.V. Avramov, Y.V. Mikhlin, E. Kurilov, Asymptotic analysis of nonlinear dynamics of simply supported cylindrical shells, Nonlinear Dynamics 47 (2007) 331-352.

[62] P.B. Gonçalves, F.M.A. Silva, Z.G. del Prado, Low-dimensional models for the nonlinear vibration analysis of cylindrical shells based on a perturbation procedure and proper orthogonal decomposition, Journal of Sound and Vibration 315 (2008) 641-663.

[63] C. Touzé, M. Amabili, O. Thomas, Reduced-order models for large-amplitude vibrations of shells including in-plane inertia, Computer Methods in Applied Mechanics and Engineering 197 (2008) 2030-2045.

[64] M. Rougui, F. Moussaoui, R. Benamar, Geometrically non-linear free and forced vibrations of simply supported circular cylindrical shells: a semianalytical approach, International Journal of Non-Linear Mechanics 42 (2007) 1102-1115

[65] K.V. Avramov, Multidimensional models of traveling waves and nonlinear modes in cylindrical shells, International Applied Mechanics 47 (2011) 70-77.

[66] K.V. Avramov, Nonlinear modes of vibrations for simply supported cylindrica shell with geometrical nonlinearity, Acta Mechanica 223 (2012) 279-292.

[67] C. Chen, L. Dai, Nonlinear vibration and stability of a rotary truncated conical shell with intercoupling of high and low order modals, Communications in Nonlinear Science and Numerical Simulation 14 (2009) 254-269.

[68] Y.Q. Wang, X.H. Guo, H.H. Chang, H.Y. Li, Nonlinear dynamic response of rotating circular cylindrical shells with precession of vibrating shape-Part I: numerical solution, International Journal of Mechanical Sciences 52 (2010) $1217-1224$.

[69] Y.Q. Wang, X.H. Guo, H.H. Chang, H.Y. Li, Nonlinear dynamic response of rotating circular cylindrical shells with precession of vibrating shape-Part II: approximate analytical solution, International Journal of Mechanical Sciences 52 (2010) 1208-1216.

[70] Y.Q. Wang, X.H. Guo, Y.G. Li, J. Li, Nonlinear traveling wave vibration of a circular cylindrical shell subjected to a moving concentrated harmonic force, Journal of Sound and Vibration 329 (2010) 338-352.

[71] Y. Liu, F. Chu, Nonlinear vibrations of rotating thin circular cylindrical shell, Nonlinear Dynamics 67 (2012) 1467-1479.

[72] C. Chen, Nonlinear dynamic of a rotating truncated conical shell, in: L. Dai, R.N. Jazar (Eds.), Nonlinear Approaches in Engineering Applications, Springer, New York, 2012, pp. 349-391.

[73] Y. Kurylov, M. Amabili, Polynomial versus trigonometric expansions for nonlinear vibrations of circular cylindrical shells with different boundary conditions, Journal of Sound and Vibration 329 (2010) 1435-1449.

[74] Y. Kurylov, M. Amabili, Nonlinear vibrations of clamped-free circular cylindrical shells, Journal of Sound and Vibration 330 (2011) 5363-5381.

[75] W. Zhang, R. Zhou, J. Zu, Nonlinear vibrations of a shell-shaped workpiece during high-speed milling process, Nonlinear Dynamics 72 (2013) 1-21.

[76] F. Bakhtiari-Nejad, S.M. Mousavi Bideleh, Nonlinear free vibration analysis of prestressed circular cylindrical shells on the Winkler/Pasternak foundation, Thin-Walled Structures 53 (2012) 26-39.

[77] V.A. Krysko, J. Awrejcewicz, N.E. Saveleva, Stability, bifurcation and chaos of closed flexible cylindrical shells, International Journal of Mechanical Sciences 50 (2008) 247-274

[78] A.V. Krysko, J. Awrejcewicz, E.S. Kuznetsova, V.A. Krysko, Chaotic vibrations of closed cylindrical shells in a temperature field, Shock and Vibration 15 (2008) 335-343.

[79] A.V. Krysko, J. Awrejcewicz, E.S. Kuznetsova, V.A. Krysko, Chaotic vibrations of closed cylindrical shells in a temperature field, International Journal of Bifurcation and Chaos 18 (2008) 1515-1529.

[80] J. Awrejcewicz, V.A. Krysko, I.V. Papkova, A.V. Krysko, Routes to chaos in continuous mechanical systems. Part 1: mathematical models and solution methods, Chaos, Solitons \& Fractals 45 (2012) 687-708. 
[81] A.V. Krysko, J. Awrejcewicz, I.V. Papkova, V.A. Krysko, Routes to chaos in continuous mechanical systems: Part 2. Modelling transitions from regular to chaotic dynamics, Chaos, Solitons \& Fractals 45 (2012) 709-720.

[82] J. Awrejcewicz, A.V. Krysko, I.V. Papkova, V.A. Krysko, Routes to chaos in continuous mechanical systems. Part 3: the Lyapunov exponents, hyper, hyper-hyper and spatial-temporal chaos, Chaos, Solitons \& Fractals 45 (2012) 721-736.

[83] A.H. Nayfeh, H.N. Arafat, Axisymmetric vibrations of closed spherical shells: equations of motion and bifurcation analysis, Structural Control and Health Monitoring 13 (2006) 388-416.

[84] F. Pellicano, M. Amabili, Stability and vibration of empty and fluid-filled circular cylindrical shells under static and periodic axial loads, International Journal of Solids and Structures 40 (2003) 3229-3251.

[85] A.A. Popov, Parametric resonance in cylindrical shells: a case study in the nonlinear vibration of structural shells, Engineering Structures 25 (2003) 789-799.

[86] P.B. Gonçalves, Z.G. del Prado, Effect of non-linear modal interaction on the dynamic instability of axially excited cylindrical shells, Computers \& Structures 82 (2004) 2621-2634.

[87] P.B. Gonçalves, Z.G. del Prado, Low-dimensional Galerkin models for nonlinear vibration and instability analysis of cylindrical shells, Nonlinear Dynamics 41 (2005) 129-145.

[88] P.B. Gonçalves, F.M.A. Da Silva, Z.G. del Prado, Transient stability of empty and fluid-filled cylindrical shells, Journal of the Brazilian Society of Mechan-ical Sciences and Engineering 28 (2006) 331-338.

[89] P. Gonçalves, F.A. Silva, Z.G. del Prado, Global stability analysis of parametrically excited cylindrical shells through the evolution of basin boundaries, Nonlinear Dynamics 50 (2007) 121-145.

[90] P.B. Gonçalves, F.M.A. Silva, Z.G. del Prado, Transient and steady state stability of cylindrical shells under harmonic axial loads, International Journal of Non-Linear Mechanics 42 (2007) 58-70.

[91] R. Kochurov, K.V. Avramov, Nonlinear modes and traveling waves of parametrically excited cylindrical shells, Journal of Sound and Vibration 329 (2010) 2193-2204.

[92] R. Kochurov, K.V. Avramov, Parametric vibration of cylindrical shells in the region of combination resonances under geometrically nonlinear deformation, Journal of Mathematical Sciences 174 (2011) 283-294.

[93] P. Gonçalves, F.A. Silva, G. Rega, S. Lenci, Global dynamics and integrity of a two-dof model of a parametrically excited cylindrical shell, Nonlinear Dynamics 63 (2011) 61-82.

[94] F. Pellicano, K.V. Avramov, Linear and nonlinear dynamics of a circular cylindrical shell connected to a rigid disk, Communications in Nonlinear Science and Numerical Simulation 12 (2007) 496-518.

[95] F. Pellicano, Vibrations of circular cylindrical shells: theory and experiments, Journal of Sound and Vibration 303 (2007) 154-170.

[96] F. Pellicano, Dynamic instability of a circular cylindrical shell carrying a top mass under base excitation: experiments and theory, International Journal of Solids and Structures 48 (2011) 408-427.

[97] V.D. Kubenko, P.S. Koval'chuk, Influence of initial geometric imperfections on the vibrations and dynamic stability of elastic shells, International Applied Mechanics 40 (2004) 847-877.

[98] G. Catellani, F. Pellicano, D. Dall'Asta, M. Amabili, Parametric instability of a circular cylindrical shell with geometric imperfections, Computers \& Structures 82 (2004) 2635-2645.

[99] F. Pellicano, M. Amabili, Dynamic instability and chaos of empty and fluidfilled circular cylindrical shells under periodic axial loads, Journal of Sound and Vibration 293 (2006) 227-252.

[100] F. Pellicano, Dynamic stability and sensitivity to geometric imperfections of strongly compressed circular cylindrical shells under dynamic axial loads, Communications in Nonlinear Science and Numerical Simulation 14 (2009) 3449-3462.

[101] R. Kochurov, K.V. Avramov, On effect of initial imperfections on parametric vibrations of cylindrical shells with geometrical non-linearity, International Journal of Solids and Structures 49 (2012) 537-545

[102] N.J. Mallon, R.H.B. Fey, H. Nijmeijer, Dynamic stability of a thin cylindrical shell with top mass subjected to harmonic base-acceleration, International Journal of Solids and Structures 45 (2008) 1587-1613.

[103] V.D. Kubenko, P.S. Koval'chuk, L.A. Kruk, On multimode nonlinear vibrations of filled cylindrical shells, International Applied Mechanics 39 (2003) 85-92.

[104] P.S. Koval'chuk, V.G. Filin, Circumferential traveling waves in filled cylindrical shells, International Applied Mechanics 39 (2003) 192-196

[105] P.S. Koval'chuk, V.G. Filin, On modes of flexural vibrations of initially bent cylindrical shells partially filled with a liquid, International Applied Mechanics 39 (2003) 464-471.

[106] V.D. Kubenko, P.S. Koval'chuk, L.A. Kruk, On free nonlinear vibrations of fluidfilled cylindrical shells with multiple natural frequencies, International Applied Mechanics 41 (2005) 1193-1203.

[107] P.S. Koval'chuk, L.A. Kruk, On the spectrum of natural frequencies of circular cylindrical shells completely filled with a fluid, International Applied Mechanics 42 (2006) 529-535.

[108] F.A. Silva, P. Gonçalves, Z.G. del Prado, An alternative procedure for the nonlinear vibration analysis of fluid-filled cylindrical shells, Nonlinear Dynamics 66 (2011) 303-333.

[109] P.S. Koval'chuk, L.A. Kruk, Chaotic modes of forced nonlinear vibrations of fluid-filled cylindrical shells, International Applied Mechanics 39 (2003) 1452 1457.
[110] K.N. Karagiozis, M. Amabili, M.P. Païdoussis, A.K. Misra, Nonlinear vibrations of fluid-filled clamped circular cylindrical shells, Journal of Fluids and Structures 21 (2005) 579-595.

[111] P.S. Koval'chuk, L.A. Kruk, The problem of forced nonlinear vibrations of cylindrical shells completely filled with liquid, International Applied Mechanics 41 (2005) 154-160.

[112] P.S. Koval'chuk, L.A. Kruk, Wave deformation modes of fluid-containing cylindrical shells under periodic force, International Applied Mechanics 41 (2005) 526-531.

[113] P.S. Koval'chuk, N.P. Podchasov, G.N. Puchka, Studying the forced vibrations of fluid-filled cylindrical shells with regard to the nonlinear interaction of different flexural modes, International Applied Mechanics 42 (2006) 928-935.

[114] V.D. Kubenko, P.S. Koval'chuk, L.A. Kruk, Nonlinear vibrations of cylindrical shells filled with a fluid and subjected to longitudinal and transverse periodic excitation, International Applied Mechanics 46 (2010) 186-194.

[115] M. Amabili, A. Sarkar, M.P. Païdoussis, Reduced-order models for nonlinear vibrations of cylindrical shells via the proper orthogonal decomposition method, Journal of Fluids and Structures 18 (2003) 227-250.

[116] M. Amabili, A. Sarkar, M.P. Païdoussis, Chaotic vibrations of circular cylindrical shells: Galerkin versus reduced-order models via the proper orthogonal decomposition method, Journal of Sound and Vibration 290 (2006) 736-762.

[117] C. Touzé, M. Amabili, Nonlinear normal modes for damped geometrically nonlinear systems: application to reduced-order modelling of harmonically forced structures, Journal of Sound and Vibration 298 (2006) 958-981.

[118] M. Amabili, C. Touzé, Reduced-order models for nonlinear vibrations of fluidfilled circular cylindrical shells: comparison of POD and asymptotic nonlinear normal modes methods, Journal of Fluids and Structures 23 (2007) 885-903.

[119] F.M.A. Silva, P.B. Gonçalves, Z.G. del Prado, Parametric instability and snapthrough of partially fluid-filled cylindrical shells, Procedia Engineering 14 (2011) 598-605.

[120] M.P. Païdoussis, Fluid-Structure Interactions: Slender Structures and Axial Flow, Elsevier Academic Press, London, UK, 2003.

[121] K.N. Karagiozis, M.P. Païdoussis, A.K. Misra, E. Grinevich, An experimental study of the nonlinear dynamics of cylindrical shells with clamped ends subjected to axial flow, Journal of Fluids and Structures 20 (2005) 801-816.

[122] K.N. Karagiozis, M.P. Païdoussis, A.K. Misra, E. Grinevich, Addendum to "an experimental study of the nonlinear dynamics of cylindrical shells with clamped ends subjected to axial flow", Journal of Fluids and Structures 22 (2006) 595-596.

[123] K.N. Karagiozis, M.P. Païdoussis, A.K. Misra, Transmural pressure effects on the stability of clamped cylindrical shells subjected to internal fluid flow: theory and experiments, International Journal of Non-Linear Mechanics 42 (2007) 13-23.

[124] K.N. Karagiozis, M.P. Païdoussis, M. Amabili, Effect of geometry on the stability of cylindrical clamped shells subjected to internal fluid flow, Computers \& Structures 85 (2007) 645-659.

[125] K.N. Karagiozis, M.P. Païdoussis, M. Amabili, A.K. Misra, Nonlinear stability of cylindrical shells subjected to axial flow: theory and experiments, Journal of Sound and Vibration 309 (2008) 637-676.

[126] M. Amabili, K. Karagiozis, M.P. Païdoussis, Effect of geometric imperfections on non-linear stability of circular cylindrical shells conveying fluid, International Journal of Non-Linear Mechanics 44 (2009) 276-289.

[127] Z. del Prado, P.B. Gonçalves, M.P. Païdoussis, Nonlinear vibrations and imperfection sensitivity of a cylindrical shell containing axial fluid flow, Journal of Sound and Vibration 327 (2009) 211-230.

[128] K. Karagiozis, M. Amabili, M.P. Païdoussis, Nonlinear dynamics of harmonically excited circular cylindrical shells containing fluid flow, Journal of Sound and Vibration 329 (2010) 3813-3834.

[129] P.S. Koval'chuk, Nonlinear vibrations of a cylindrical shell containing a flowing fluid, International Applied Mechanics 41 (2005) 405-412.

[130] P.S. Koval'chuk, L.A. Kruk, Forced nonlinear oscillations of cylindrical shells interacting with fluid flow, International Applied Mechanics 42 (2006) 447-454

[131] V.D. Kubenko, P.S. Koval'chuk, L.A. Kruk, Application of asymptotic methods to the investigation of one-frequency nonlinear oscillations of cylindrical shells interacting with moving fluid, Ukrainian Mathematical Journal 59 (2007) 533 545.

[132] V.D. Kubenko, P.S. Koval'chuk, N.P. Podchasov, Analysis of nonstationary processes in cylindrical shells interacting with a fluid flow, International Applied Mechanics 46 (2011) 1119-1131.

[133] M. Paak, M.P. Païdoussis, A.K. Misra, Nonlinear dynamics and stability of cantilevered circular cylindrical shells conveying fluid, Journal of Sound and Vibration 332 (2013) 3474-3498.

[134] I. Chueshov, I. Ryzhkova, Unsteady interaction of a viscous fluid with an elastic shell modeled by full von Karman equations, Journal of Differential Equations 254 (2013) 1833-1862.

[135] E.A. Kurilov, Y.V. Mikhlin, Nonlinear vibrations of cylindrical shells with initial imperfections in a supersonic flow, International Applied Mechanics 43 (2007) 1000-1008.

[136] E.L. Jansen, Analytical-numerical models for flutter of cylindrical shells in supersonic flow, in: K.J. Bathe (Ed.), Computational Fluid and Solid Mechanics 2003, Elsevier Science Ltd., Oxford, 2003, pp. 1377-1380.

[137] M. Amabili, F. Pellicano, Non-linear supersonic flutter of circular cylindrical shells, AIAA Journal 39 (2001) 564-573.

[138] M. Amabili, F. Pellicano, Multimode appraoch to nonlinear supersonic flutter of imperfect circular cylindrical shells, Journal of Applied Mechanics 69 (2002) 117-129. 
[139] C. Sansour, A time integration scheme with energy-momentum conservation for a shell formulation with arbitrary geometric and material non-linearities, Computers \& Structures 82 (2004) 2753-2763.

[140] J. Awrejcewicz, V.A. Krysko, Nonlinear coupled problems in dynamics of shells, International Journal of Engineering Science 41 (2003) 587-607.

[141] S. Xue-feng, L. Jiao-xia, W. Yong-zhong, Analysis of thermal-elastic coupling vibration of large deflection cylindrical shell, Applied Mathematics and Mechanics 25 (2004) 994-1000.

[142] N.A. Shul'ga, S.Y. Bogdanov, Forced axisymmetric nonlinear vibrations of reinforced conical shells, International Applied Mechanics 39 (2003) 1447-1451.

[143] N.A. Shul'ga, S.Y. Bogdanov, On the deformation of a ribbed cylindrical shell with initial deflection under dynamic loading, International Applied Mechanics 41 (2005) 1378-1383.

[144] V.F. Meish, A.S. Kairov, Vibrations of reinforced cylindrical shells with initial deflections under nonstationary loads, International Applied Mechanics 41 (2005) 42-48.

[145] P.Z. Lugovoi, V.F. Meish, B.P. Rybakin, G.V. Sekrieru, On numerical solution of dynamic problems in the theory of reinforced shells, International Applied Mechanics 42 (2006) 536-540.

[146] P.Z. Lugovoi, V.F. Meish, B.P. Rybakin, G.V. Sekrieru, Dynamics of reinforced compound shells under nonstationary loads, International Applied Mechanics 42 (2006) 455-460.

[147] P.Z. Lugovoi, V.F. Meish, K.G. Golovko, Solving axisymmetric dynamic problems for reinforced shells of revolution on an elastic foundation, International Applied Mechanics 45 (2009) 193-199.

[148] M.X. Shi, Q.M. Li, Instability in the transformation between extensional and flexural modes in thin-walled cylindrical shells, European Journal of Mechanics - A/Solids 30 (2011) 33-45.

[149] J. Yang, X. Yang, J. Turner, Nonlinear vibrations of electroelastic shells with relatively large shear deformations, Science in China Series G: Physics, Mechanics and Astronomy 49 (2006) 660-670.

[150] V.G. Karnaukhov, Y.V. Tkachenko, Studying the harmonic vibrations of a cylindrical shell made of a nonlinear elastic piezoelectric material, International Applied Mechanics 44 (2008) 442-447.

[151] H. Yong, X. He, Y. Zhou, Dynamics of a thick-walled dielectric elastome spherical shell, International Journal of Engineering Science 49 (2011) 792-800.

[152] X. He, H. Yong, Y. Zhou, The dynamics response and stability of a dielectric elastomer cylindrical shell under static and periodic voltage, Guti Lixue Xuebao/Acta Mechanica Solida Sinica 33 (2012) 341-348.

[153] Q. Dong, Q.M. Li, J.Y. Zheng, Further study on strain growth in spherical containment vessels subjected to internal blast loading, International Journal of Impact Engineering 37 (2010) 196-206.

[154] B.K. Eshmatov, Nonlinear vibrations of viscoelastic cylindrical shells taking into account shear deformation and rotatory inertia, Nonlinear Dynamics 50 (2007) 353-361.

[155] B. Eshmatov, Dynamic stability of viscoelastic circular cylindrical shells taking into account shear deformation and rotatory inertia, Applied Mathematics and Mechanics 28 (2007) 1319-1330.

[156] B.K. Eshmatov, Nonlinear vibrations and dynamic stability of a viscoelastic circular cylindrical shell with shear strain and inertia of rotation taken into account, Mechanics of Solids 44 (2009) 421-434.

[157] M. Amabili, M. Pellegrini, M. Tommesani, Experiments on large-amplitude vibrations of a circular cylindrical panel, Journal of Sound and Vibration 260 (2003) 537-547.

[158] M. Amabili, Nonlinear vibrations of circular cylindrical panels, Journal of Sound and Vibration 281 (2005) 509-535.

[159] M. Amabili, Non-linear vibrations of doubly curved shallow shells, International Journal of Non-Linear Mechanics 40 (2005) 683-710.

[160] M. Amabili, Theory and experiments for large-amplitude vibrations of circular cylindrical panels with geometric imperfections, Journal of Sound and Vibration 298 (2006) 43-72.

[161] M. Amabili, Effect of boundary conditions on nonlinear vibrations of circular cylindrical panels, Journal of Applied Mechanics 74 (2007) 645-657.

[162] C. Sansour, P. Wriggers, J. Sansour, A finite element post-processed Galerkin method for dimensional reduction in the non-linear dynamics of solids, Applications to shells, Computational Mechanics 32 (2003) 104-114

[163] P. Ribeiro, A hierarchical finite element for geometrically non-linear vibration of doubly curved, moderately thick isotropic shallow shells, International Journal for Numerical Methods in Engineering 56 (2003) 715-738.

[164] A.R. de Faria, Finite element analysis of the dynamic response of cylindrical panels under traversing loads, European Journal of Mechanics - A/Solids 23 (2004) 677-687.

[165] A. Przekop, M.S. Azzouz, X. Guo, C. Mei, L. Azrar, Finite element multiplemode approach to nonlinear free vibrations of shallow shells, AIAA Journal 42 (2004) 2373-2381.

[166] P. Ribeiro, Non-linear free periodic vibrations of open cylindrical shallow shells, Journal of Sound and Vibration 313 (2008) 224-245.

[167] P. Ribeiro, Forced large amplitude periodic vibrations of cylindrical shallow shells, Finite Elements in Analysis and Design 44 (2008) 657-674

[168] P. Ribeiro, B. Cochelin, S. Bellizzi, Non-linear vibrations of deep cylindrical shells by the $p$-version finite element method, Shock and Vibration 17 (2010) 21-37.

[169] F. Alijani, M. Amabili, F. Bakhtiari-Nejad, On the accuracy of the multiple scales method for non-linear vibrations of doubly curved shallow shells, International Journal of Non-Linear Mechanics 46 (2011) 170-179.
[170] L. Kurpa, G. Pilgun, M. Amabili, Nonlinear vibrations of shallow shells with complex boundary: R-functions method and experiments, Journal of Sound and Vibration 306 (2007) 580-600.

[171] I.D. Breslavsky, K.V. Avramov, Vibrations of a complex-shaped panel, International Applied Mechanics 46 (2010) 580-587.

[172] I.D. Breslavsky, K.V. Avramov, Nonlinear modes of cylindrical panels with complex boundaries. R-Function method, Meccanica 46 (2011) 817-832.

[173] I.D. Breslavsky, E.A. Strel'Nikova, K.V. Avramov, Free vibrations of a shallow shell in fluid under geometrically nonlinear deformation, Strength of Materials 43 (2011) 25-32.

[174] I.D. Breslavsky, E.A. Strel'Nikova, K.V. Avramov, Dynamics of shallow shells with geometrical nonlinearity interacting with fluid, Computers and Structures 89 (2011) 496-506.

[175] G. Pilgun, M. Amabili, Non-linear vibrations of shallow circular cylindrical panels with complex geometry. Meshless discretization with the R-functions method, International Journal of Non-Linear Mechanics 47 (2012) 137-152

[176] C. Sansour, P. Wriggers, J. Sansour, On the design of energy-momentum integration schemes for arbitrary continuum formulations. Applications to classical and chaotic motion of shells, International Journal for Numerical Methods in Engineering 60 (2004) 2419-2440.

[177] K. Nagai, S. Maruyama, M. Oya, T. Yamaguchi, Chaotic oscillations of a shallow cylindrical shell with a concentrated mass under periodic excitation, Computers \& Structures 82 (2004) 2607-2619.

[178] V.A. Krysko, J. Awrejcewicz, T.V. Shchekaturova, Chaotic vibrations of spherical and conical axially symmetric shells, Archive of Applied Mechanics 74 (2005) 338-358.

[179] J. Awrejcewicz, V.A. Krysko, T.V. Shchekaturova, Transitions from regular to chaotic vibrations of spherical and conical axially-symmetric shells, International Journal of Structural Stability and Dynamics 5 (2005) 359-385.

[180] K. Nagai, S. Maruyama, T. Murata, T. Yamaguchi, Experiments and analysis on chaotic vibrations of a shallow cylindrical shell-panel, Journal of Sound and Vibration 305 (2007) 492-520.

[181] X.Z. Wang, C.X. Liang, M.J. Han, K.Y. Yeh, G. Wang, Nonlinear dynamical behavior of shallow cylindrical reticulated shells, Applied Mathematics and Mechanics (English Edition) 28 (2007) 151-156.

[182] J. Awrejcewicz, V.A. Krysko, V. Nazar'iantz, Chaotic vibrations of flexible infinite length cylindrical panels using the Kirchhoff-Love model, Communications in Nonlinear Science and Numerical Simulation 12 (2007) 519-542.

[183] S. Maruyama, K. Nagai, Y. Tsuruta, Modal interaction in chaotic vibrations of a shallow double-curved shell-panel, Journal of Sound and Vibration 315 (2008) 607-625

[184] K. Nagai, S. Maruyama, K. Hasegawa, T. Yamaguchi, Experiments on chaotic vibrations of a shallow cylindrical rectangular-panel with clamped edges at opposite boundaries, Nihon Kikai Gakkai Ronbunshu, C Hen/Transactions of the Japan Society of Mechanical Engineers, Part C 75 (2009) 1264-1269.

[185] B.A. Khudayarov, N.G. Bandurin, Numerical investigation of nonlinear vibrations of viscoelastic plates and cylindrical panels in a gas flow, Journal of Applied Mechanics and Technical Physics 48 (2007) 279-284.

[186] B.K. Eshmatov, D.A. Khodzhaev, Non-linear vibration and dynamic stability of a viscoelastic cylindrical panel with concentrated mass, Acta Mechanica 190 (2007) 165-183

[187] B.K. Eshmatov, D.A. Khodzhaev, Dynamic stability of a viscoelastic cylindrical panel with concentrated masses, Strength of Materials 40 (2008) 491-502.

[188] V.G. Karnaukhov, Y.V. Tkachenko, Active damping of the resonant vibrations of a flexible cylindrical panel with sensors and actuators, International Applied Mechanics 47 (2011) 720-726.

[189] G. Odeh, Nonlinear dynamics of shallow spherical caps subjected to peripheral loading, Nonlinear Dynamics 33 (2003) 155-164.

[190] O. Thomas, C. Touzé, A. Chaigne, Non-linear vibrations of free-edge thin spherical shells: modal interaction rules and 1:1:2 internal resonance, International Journal of Solids and Structures 42 (2005) 3339-3373.

[191] C. Touzé, O. Thomas, Non-linear behaviour of free-edge shallow spherical shells: effect of the geometry, International Journal of Non-Linear Mechanics 41 (2006) 678-692.

[192] O. Thomas, C. Touzé, É. Luminais, Non-linear vibrations of free-edge thin spherical shells: experiments on a 1:1:2 internal resonance, Nonlinear Dynamics 49 (2007) 259-284.

[193] C. Camier, C. Touzé, O. Thomas, Non-linear vibrations of imperfect free-edge circular plates and shells, European Journal of Mechanics - A/Solids 28 (2009) 500-515.

[194] H. Yuan, R.H. Liu, Nonlinear vibration of corrugated shallow shells under uniform load, Applied Mathematics and Mechanics (English Edition) 28 (2007) 573-580.

[195] H. Yuan, Method of Green's function of nonlinear vibration of corrugated shallow shells, Science in China, Series G: Physics, Mechanics and Astronomy 51 (2008) 678-686.

[196] Y.G. Wang, H.F. Song, On the nonlinear vibration of heated bimetallic shallow shells of revolution, International Journal of Mechanical Sciences 52 (2010) 464-470.

[197] M.S. Soliman, P.B. Gonçalves, Chaotic behavior resulting in transient and steady state instabilities of pressure-loaded shallow spherical shells, Journal of Sound and Vibration 259 (2003) 497-512.

[198] A.V. Krysko, J. Awrejcewicz, I.V. Papkova, Chaotic vibrations of sector-type spherical shells, Journal of Computational and Nonlinear Dynamics 3 (2008) 041005 (17 pp). 
[199] A. Chaigne, C. Touzé, O. Thomas, Nonlinear vibrations and chaos in gongs and cymbals, Acoustical Science and Technology 26 (2005) 403-409.

[200] S. Bilbao, Percussion synthesis based on models of nonlinear shell vibration, IEEE Transactions on Audio, Speech and Language Processing 18 (2010) 872-880.

[201] N. Quaegebeur, A. Chaigne, G. Lemarquand, Transient modal radiation of axisymmetric sources: application to loudspeakers, Applied Acoustics 71 (2010) 335-350.

[202] Y.G. Zhao, X.Z. Wang, K.Y. Ye, Nonlinear vibration of thin shallow conic shells under combined action of peripheral moment and transverse loads, Applied Mathematics and Mechanics (English Edition) 24 (2003) 1381-1389.

[203] Y.G. Wang, S.L. Dai, Thermoelastically coupled axisymmetric nonlinea vibration of shallow spherical and conical shells, Applied Mathematics and Mechanics (English Edition) 25 (2004) 430-439.

[204] X.Z. Wang, M.J. Han, Y.G. Zhao, K.Y. Yeh, Nonlinear natural frequency of shallow conical shells with variable thickness, Applied Mathematics and Mechanics (English Edition) 26 (2005) 277-282.

[205] X.Z. Wang, M.J. Han, Y.Y. Zhao, Y.G. Zhao, Nonlinear dynamical bifurcation and chaotic motion of shallow conical lattice shell, Applied Mathematics and Mechanics (English Edition) 27 (2006) 661-666.

[206] V.F. Meish, Numerical solution of dynamic problems for reinforced ellipsoidal shells under nonstationary loads, International Applied Mechanics 41 (2005) 386-391.

[207] V.F. Meish, N.V. Maiborodina, Nonaxisymmetric vibrations of ellipsoidal shells under nonstationary distributed loads, International Applied Mechanics 44 (2008) 1015-1024.

[208] V.F. Meish, N.V. Maiborodina, Analysis of the nonaxisymmetric vibrations of flexible ellipsoidal shells discretely reinforced with transverse ribs under nonstationary loads, International Applied Mechanics 44 (2008) 1128-1136.

[209] M.H. Toorani, Dynamics of the geometrically non-linear analysis of anisotropic laminated cylindrical shells, International Journal of Non-Linear Mechanics 38 (2003) 1315-1335.

[210] M.H. Toorani, A.A. Lakis, Large amplitude vibrations of anisotropic cylindrical shells, Computers \& Structures 82 (2004) 2015-2025.

[211] E.L. Jansen, A comparison of analytical-numerical models for nonlinear vibrations of cylindrical shells, Computers \& Structures 82 (2004) 2647-2658.

[212] E.L. Jansen, The effect of geometric imperfections on the vibrations of anisotropic cylindrical shells, Thin-Walled Structures 45 (2007) 274-282.

[213] E.L. Jansen, The effect of static loading and imperfections on the nonlinear vibrations of laminated cylindrical shells, Journal of Sound and Vibration 315 (2008) 1035-1046

[214] E.L. Jansen, A perturbation method for nonlinear vibrations of imperfect structures: application to cylindrical shell vibrations, International Journal of Solids and Structures 45 (2008) 1124-1145.

[215] E.L. Jansen, Effect of boundary conditions on nonlinear vibration and flutter of laminated cylindrical shells, Journal of Vibration and Acoustics, Transactions of the ASME 130 (2008) 011003 (8 pp).

[216] T. Rahman, E.L. Jansen, P. Tiso, A finite element-based perturbation method for nonlinear free vibration analysis of composite cylindrical shells, International Journal of Structural Stability and Dynamics 11 (2011) 717-734.

[217] M. Amabili, Internal resonances in non-linear vibrations of a laminated circular cylindrical shell, Nonlinear Dynamics 69 (2012) 755-770.

[218] M. Amabili, Nonlinear vibrations of angle-ply laminated circular cylindrical shells: Skewed modes, Composite Structures 94 (2012) 3697-3709.

[219] M. Amabili, Reduced-order models for nonlinear vibrations, based on natural modes: the case of the circular cylindrical shell, Philosophical Transactions of the Royal Society A 371 (2013) 20120474.

[220] H.S. Shen, Boundary layer theory for the nonlinear vibration of anisotropic laminated cylindrical shells, Composite Structures 97 (2013) 338-352.

[221] E.L. Jansen, Dynamic stability problems of anisotropic cylindrical shells via a simplified analysis, Nonlinear Dynamics 39 (2005) 349-367.

[222] V.D. Kubenko, P.S. Koval'Chuk, Experimental studies of the vibrations and dynamic stability of laminated composite shells, International Applied Mechanics 45 (2009) 514-533.

[223] N.J. Mallon, Dynamic Stability of Thin-walled Structures. A Semi-analytical and Experimental Approach (Ph.D. thesis), Eindhoven University of Technology Library, ISBN 978-90-386-1374-1, 2008.

[224] N.J. Mallon, R.H.B. Fey, H. Nijmeijer, Dynamic stability of a base-excited thin orthotropic cylindrical shell with top mass: simulations and experiments, Journal of Sound and Vibration 329 (2010) 3149-3170.

[225] E.I. Bespalova, G.P. Urusova, Identifying the domains of dynamic instability for inhomogeneous shell systems under periodic loads, International Applied Mechanics 47 (2011) 186-194.

[226] T. Rahman, E.L. Jansen, Z. Gürdal, Dynamic buckling analysis of composite cylindrical shells using a finite element based perturbation method, Nonlinear Dynamics 66 (2011) 389-401.

[227] M. Shariyat, Nonlinear thermomechanical dynamic buckling analysis of imperfect viscoelastic composite/sandwich shells by a doublesuperposition global-local theory and various constitutive models, Composite Structures 93 (2011) 2833-2843.

[228] M. Shariyat, Non-linear dynamic thermo-mechanical buckling analysis of the imperfect laminated and sandwich cylindrical shells based on a global-local theory inherently suitable for non-linear analyses, International Journal of Non-Linear Mechanics 46 (2011) 253-271.
[229] P.Z. Lugovoi, V.F. Meish, S.E. Shtantsel, Forced nonstationary vibrations of a sandwich cylindrical shell with cross-ribbed core, International Applied Mechanics 41 (2005) 161-167.

[230] V. Dogan, R. Vaicaitis, Nonlinear response of double-wall cylindrical shell vibrations under random excitation, Journal of Aerospace Engineering 19 (2006) 46-54.

[231] V.A. Zarutskii, P.Z. Lugovoi, V.F. Meish, Dynamic problems for and stressstrain state of inhomogeneous shell structures under stationary and nonstationary loads, International Applied Mechanics 45 (2009) 245-271.

[232] F. Mohammadi, R. Sedaghati, Linear and nonlinear vibration analysis of sandwich cylindrical shell with constrained viscoelastic core layer, International Journal of Mechanical Sciences 54 (2012) 156-171.

[233] K. Dong, X. Wang, Influences of large deformation and rotary inertia on wave propagation in piezoelectric cylindrically laminated shells in thermal environment, International Journal of Solids and Structures 43 (2006) 1710-1726

[234] K. Dong, X. Wang, Wave propagation characteristics in piezoelectric cylindrical laminated shells under large deformation, Composite Structures 77 (2007) 171-181.

[235] V.I. Kozlov, T.V. Karnaukhova, M.V. Peresun'ko, Numerical modeling of the active damping of forced thermomechanical resonance vibrations of viscoelastic shells of revolution with the help of piezoelectric inclusions, Journal of Mathematical Sciences 171 (2010) 565-578.

[236] Y.Y. Lee, C.F. Ng, X. Guo, Nonlinear random response of cylindrical panels to acoustic excitations using finite element modal method, Nonlinear Dynamics 31 (2003) 327-345.

[237] N.V.S. Naidu, P.K. Sinha, Nonlinear free vibration analysis of laminated composite shells in hygrothermal environments, Composite Structures 77 (2007) 475-483.

[238] N. Nanda, J.N. Bandyopadhyay, Nonlinear free vibration analysis of laminated composite cylindrical shells with cutouts, Journal of Reinforced Plastics and Composites 26 (2007) 1413-1427.

[239] N. Nanda, J.N. Bandyopadhyay, Large amplitude free vibration of laminated composite shells with cutout, Aircraft Engineering and Aerospace Technology 80 (2008) 165-174

240] S.K. Panda, B.N. Singh, Non-linear free vibration analysis of laminated composite cylindrical/hyperboloid shell panels based on higher-order shea deformation theory using non-linear finite-element method, Proceedings of the Institution of Mechanical Engineers, Part G: Journal of Aerospace Engineering 222 (2008) 993-1006.

[241] S.K. Panda, B.N. Singh, Nonlinear free vibration of spherical shell panel using higher order shear deformation theory - a finite element approach, International Journal of Pressure Vessels and Piping 86 (2009) 373-383.

[242] S.K. Panda, B.N. Singh, Nonlinear free vibration analysis of thermally postbuckled composite spherical shell panel, International Journal of Mechanics and Materials in Design 6 (2010) 175-188.

[243] A.H. Hashemian, J.E. Jam, Nonlinear free dynamic response of laminated compressible cylindrical shell panels, Mechanics of Composite Materials 46 (2010) 15-28.

[244] N. Nanda, Non-linear free and forced vibrations of piezoelectric laminated shells in thermal environments, IES Journal Part A: Civil and Structural Engineering 3 (2010) 147-160.

[245] N. Nanda, S. Pradyumna, Nonlinear dynamic response of laminated shells with imperfections in hygrothermal environments, Journal of Composite Materials 45 (2011) 2103-2112.

[246] S.K. Panda, B.N. Singh, Large amplitude free vibration analysis of thermally post-buckled composite doubly curved panel using nonlinear FEM, Finite Elements in Analysis and Design 47 (2011) 378-386.

[247] F. Mohammadi, R. Sedaghati, Nonlinear free vibration analysis of sandwich shell structures with a constrained electrorheological fluid layer, Smart Materials and Structures 21 (2012) 075035 (18 pp).

248] S.K. Panda, B.N. Singh, Nonlinear finite element analysis of thermal postbuckling vibration of laminated composite shell panel embedded with SMA fibre, Aerospace Science and Technology 29 (2013) 47-57.

[249] L.V. Kurpa, Nonlinear free vibrations of multilayer shallow shells with a symmetric structure and with a complicated form of the plan, Journal of Mathematical Sciences 162 (2009) 85-98.

[250] J. Awrejcewicz, L. Kurpa, T. Shmatko, Large amplitude free vibration of orthotropic shallow shells of complex shapes with variable thickness, Latin American Journal of Solids and Structures 10 (2013) 149-162.

[251] A.A. Yazdi, Applicability of homotopy perturbation method to study the nonlinear vibration of doubly curved cross-ply shells, Composite Structures 96 (2013) 526-531.

[252] M. Amabili, S. Carra, R. Garziera, M. Pellegrini, Experiments on nonlinear vibrations of graphite/epoxy composite curved panels, Journal of the Mechanical Behavior of Materials 16 (2005) 287-296.

[253] C. Adam, Nonlinear flexural vibrations of layered panels with initial imperfections, Acta Mechanica 181 (2006) 91-104.

[254] C. Adam, Moderately large vibrations of doubly curved shallow open shells composed of thick layers, Journal of Sound and Vibration 299 (2007) 854-868.

[255] A. Abe, Y. Kobayashi, G. Yamada, Nonlinear dynamic behaviors of clamped laminated shallow shells with one-to-one internal resonance, Journal of Sound and Vibration 304 (2007) 957-968.

[256] P. Ribeiro, On the influence of membrane inertia and shear deformation on the geometrically non-linear vibrations of open, cylindrical, laminated clamped shells, Composites Science and Technology 69 (2009) 176-185. 
[257] N.A. Shul'ga, V.F. Meish, Forced vibration of three-layered spherical and ellipsoidal shells under axisymmetric loads, Mechanics of Composite Materials 39 (2003) 439-446.

[258] S. Lentzen, P. Kłosowski, R. Schmidt, Geometrically nonlinear finite element simulation of smart piezolaminated plates and shells, Smart Materials and Structures 16 (2007) 2265-2274.

[259] P. Ribeiro, E. Jansen, Non-linear vibrations of laminated cylindrical shallow shells under thermomechanical loading, Journal of Sound and Vibration 315 (2008) 626-640.

[260] N. Nanda, J.N. Bandyopadhyay, Nonlinear transient response of laminated composite shells, Journal of Engineering Mechanics 134 (2008) 983-990.

[261] R. Li, G.A. Kardomateas, G.J. Simitses, Nonlinear response of a shallow sandwich shell with compressible core to blast loading, Journal of Applied Mechanics, Transactions ASME 75 (2008) 0610231-06102310.

[262] Y. Fu, Z. Gao, F. Zhu, Analysis of nonlinear dynamic response and dynamic buckling for laminated shallow spherical thick shells with damage, Nonlinear Dynamics 54 (2008) 333-343.

[263] S. Pradyumna, A. Gupta, Nonlinear dynamic stability of laminated composite shells integrated with piezoelectric layers in thermal environment, Acta Mechanica 218 (2011) 295-308.

[264] S.K. Sarangi, M.C. Ray, Active damping of geometrically nonlinear vibrations of laminated composite shallow shells using vertically/obliquely reinforced 1-3 piezoelectric composites, International Journal of Mechanics and Materials in Design 7 (2011) 29-44.

[265] S.K. Sarangi, M.C. Ray, Active damping of geometrically nonlinear vibrations of doubly curved laminated composite shells, Composite Structures 93 (2011) $3216-3228$.

[266] V.G. Karnaukhov, V.I. Kozlov, T.V. Karnaukhova, Influence of dissipative heating on active damping of forced resonance vibrations of a flexible viscoelastic cylindrical panel by piezoelectric actuators, Journal of Mathematical Sciences (United States) 183 (2012) 205-221.

[267] J. Shivakumar, M.H. Ashok, M.C. Ray, Active control of geometrically nonlinear transient vibrations of laminated composite cylindrical panels using piezoelectric fiber reinforced composite, Acta Mechanica 224 (2013) 1-15.[268]

Z. del Prado, P.B. Gonçalves, M.P. Païdoussis, Non-linear vibrations and instabilities of orthotropic cylindrical shells with internal flowing fluid, International Journal of Mechanical Sciences 52 (2010) 1437-1457.

[269] V.D. Lakiza, Dynamic deformation of a cylindrical composite shell with filler subject to radial two-frequency excitation, International Applied Mechanics 46 (2011) 1386-1392.

[270] F.-M. Li, G. Yao, 1/3 Subharmonic resonance of a nonlinear composite laminated cylindrical shell in subsonic air flow, Composite Structures 100 (2013) 249-256.

[271] I.K. Oh, I. Lee, Supersonic flutter suppression of piezolaminated cylindrical panels based on multifield layerwise theory, Journal of Sound and Vibration 291 (2006) 1186-1201.

[272] W.H. Shin, I.K. Oh, I. Lee, Nonlinear flutter of aerothermally buckled composite shells with damping treatments, Journal of Sound and Vibration 324 (2009) 556-569.

[273] M. Amabili, K. Karazis, R. Mongrain, M.P. Païdoussis, R. Cartier, A three-layer model for buckling of a human aortic segment under specific flow-pressure conditions, International Journal for Numerical Methods in Biomedical Engineering 28 (2012) 495-512.

[274] M. Darabi, M. Darvizeh, A. Darvizeh, Non-linear analysis of dynamic stability for functionally graded cylindrical shells under periodic axial loading, Composite Structures 83 (2008) 201-211.

[275] S. Mahmoudkhani, H.M. Navazi, H. Haddadpour, An analytical study of the non-linear vibrations of cylindrical shells, International Journal of Non-Linear Mechanics 46 (2011) 1361-1372.

[276] H.S. Shen, Nonlinear vibration of shear deformable FGM cylindrical shells surrounded by an elastic medium, Composite Structures 94 (2012) 1144-1154.

[277] H.S. Shen, Y. Xiang, Nonlinear vibration of nanotube-reinforced composite cylindrical shells in thermal environments, Computer Methods in Applied Mechanics and Engineering 213-216 (2012) 196-205

[278] D.H. Bich, N. Xuan Nguyen, Nonlinear vibration of functionally graded circular cylindrical shells based on improved Donnell equations, Journal of Sound and Vibration 331 (2012) 5488-5501.

[279] Y.X. Hao, W. Zhang, J. Yang, Nonlinear dynamics of cantilever FGM cylindrical shell under 1:2 internal resonance relations, Mechanics of Advanced Materials and Structures 20 (2012) 819-833.

[280] Y.X. Hao, W. Zhang, L. Yang, J.H. Wang, Dynamic response of cantilever FGM cylindrical shell, Applied Mechanics and Materials 130-134 (2011) 3986-3993.

[281] W. Zhang, Y.X. Hao, J. Yang, Nonlinear dynamics of FGM circular cylindrical shell with clamped-clamped edges, Composite Structures 94 (2012) 1075-1086.

[282] G.G. Sheng, X. Wang, An analytical study of the non-linear vibrations of functionally graded cylindrical shells subjected to thermal and axial loads, Composite Structures 97 (2013) 261-268.

[283] M. Rafiee, M. Mohammadi, B. Sobhani Aragh, H. Yaghoobi, Nonlinear free and forced thermo-electro-aero-elastic vibration and dynamic response of piezoelectric functionally graded laminated composite shells, Part I: theory and analytical solutions, Composite Structures 103 (2013) 179-187.

[284] M. Rafiee, M. Mohammadi, B. Sobhani Aragh, H. Yaghoobi, Nonlinear free and forced thermo-electro-aero-elastic vibration and dynamic response of piezoelectric functionally graded laminated composite shells: Part II: numerical results, Composite Structures 103 (2013) 188-196.
[285] A.M. Najafov, A.H. Sofiyev, The non-linear dynamics of FGM truncated conical shells surrounded by an elastic medium, International Journal of Mechanical Sciences 66 (2013) 33-44.

[286] A. Deniz, A.H. Sofiyev, The nonlinear dynamic buckling response of functionally graded truncated conical shells, Journal of Sound and Vibration 332 (2013) 978-992.

[287] M. Strozzi, F. Pellicano, Nonlinear vibrations of functionally graded cylindrical shells, Thin-Walled Structures 67 (2013) 63-77.

[288] K.M. Liew, J. Yang, Y.F. Wu, Nonlinear vibration of a coating-FGM-substrate cylindrical panel subjected to a temperature gradient, Computer Methods in Applied Mechanics and Engineering 195 (2006) 1007-1026.

[289] S. Pradyumna, N. Nanda, J.N. Bandyopadhyay, Geometrically nonlinear transient analysis of functionally graded shell panels using a higher-order finite element formulation, Journal of Mechanical Engineering Research 2 (2010) 3951.

[290] S. Pradyumna, N. Nanda, Geometrically nonlinear transient response of functionally graded shell panels with initial geometric imperfection, Mechanics of Advanced Materials and Structures 20 (2013) 217-226.

[291] S.M. Chorfi, A. Houmat, Non-linear free vibration of a functionally graded doubly-curved shallow shell of elliptical plan-form, Composite Structures 92 (2010) 2573-2581.

[292] F. Alijani, M. Amabili, K. Karagiozis, F. Bakhtiari-Nejad, Nonlinear vibrations of functionally graded doubly curved shallow shells, Journal of Sound and Vibration 330 (2011) 1432-1454.

[293] F. Alijani, M. Amabili, F. Bakhtiari-Nejad, Thermal effects on nonlinear vibrations of functionally graded doubly curved shells using higher order shear deformation theory, Composite Structures 93 (2011) 2541-2553.

[294] F. Alijani, M. Amabili, Chaotic vibrations in functionally graded doubly curved shells with internal resonance, International Journal of Structural Stability and Dynamics 12 (2012) 1250047 (22 pp).

[295] D.H. Bich, D.V. Dung, V.H. Nam, Nonlinear dynamical analysis of eccentrically stiffened functionally graded cylindrical panels, Composite Structures 94 (2012) 2465-2473.

[296] D.H. Bich, D.V. Dung, V.H. Nam, Nonlinear dynamic analysis of eccentrically stiffened imperfect functionally graded doubly curved thin shallow shells, Composite Structures 96 (2013) 384-395

[297] N.D. Duc, Nonlinear dynamic response of imperfect eccentrically stiffened FGM double curved shallow shells on elastic foundation, Composite Structures 99 (2013) 88-96.

[298] R.W. Ogden, Non-linear Elastic Deformations, Dover Publications, New-York, USA, 1997.

[299] J.K. Knowles, Large amplitude oscillations of a tube of incompressible elastic material, Quarterly of Applied Mathematics 18 (1960) 71-77.

[300] J.K. Knowles, On a class of oscillations in the finite deformation theory of elasticity, Journal of Applied Mechanics 29 (1962) 283-286.

[301] M. Shahinpoor, J.L. Nowinski, Exact solution to the problem of forced large amplitude radial oscillations of a thin hyperelastic tube, International Journal of Non-Linear Mechanics 6 (1971) 193-207.

[302] M. Shahinpoor, R. Balakrishnan, Large amplitude oscillations of thick hyperelastic cylindrical shells, International Journal of Non-Linear Mechanics 13 (1978) 295-301.

[303] A.S.D. Wang, A. Ertepinar, Stability and vibrations of elastic thick-walled cylindrical and spherical shells subjected to pressure, International Journal of Non-Linear Mechanics 7 (1972) 539-555.

[304] A. Ertepinar, H.U. Akay, Radial oscillations of nonhomogeneous, thick-walled cylindrical and spherical shells subjected to finite deformations, Interna-tional Journal of Solids and Structures 12 (1976) 517-524.

[305] C. Calderer, The dynamical behaviour of nonlinear elastic spherical shells, Journal of Elasticity 13 (1983) 17-47.

[306] U. Akyüz, A. Ertepinar, Stability and asymmetric vibrations of pressurized compressible hyperelastic cylindrical shells, International Journal of NonLinear Mechanics 34 (1998) 391-404.

[307] Y. Zhu, X.Y. Luo, R.W. Ogden, Asymmetric bifurcations of thick-walled circular cylindrical elastic tubes under axial loading and external pressure, International Journal of Solids and Structures 45 (2008) 3410-3429.

[308] Y. Zhu, X.Y. Luo, R.W. Ogden, Nonlinear axisymmetric deformations of an elastic tube under external pressure, European Journal of Mechanics - A/ Solids 29 (2010) 216-229.

[309] Y. Zhu, X.Y. Luo, H.M. Wang, R.W. Ogden, C. Berry, Three-dimensional nonlinear buckling of thick-walled elastic tubes under pressure, International Journal of Non-Linear Mechanics 48 (2013) 1-14.

[310] X. Yuan, R. Zhang, H. Zhang, Controllability conditions of finite oscillations of hyperelastic cylindrical tubes composed of a class of Ogden material models, Computers, Materials and Continua 7 (2008) 155-166.

[311] J.-s. Ren, Dynamical response of hyper-elastic cylindrical shells unde periodic load, Applied Mathematics and Mechanics 29 (2008) 1319-1327.

[312] J.-s. Ren, Dynamics and destruction of internally pressurized incompressible hyper-elastic spherical shells, International Journal of Engineering Science 47 (2009) 745-753.

[313] V.D. Lakiza, Features of the motion of a gas-liquid medium in a compound shell (sphere and truncated cone) subject to vibration, International Applied Mechanics 41 (2005) 85-89.

[314] V.D. Lakiza, Dynamics of an elastic cylindrical shell with a gas-liquid medium subject to two-frequency vibrational excitation, International Applied Mechanics 44 (2008) 1294-1301. 